\title{
ARTICLE OPEN XBP1 regulates the protumoral function of tumor-associated macrophages in human colorectal cancer
}

\author{
Yahui Zhao ${ }^{1}$, Weina Zhang ${ }^{1}$, Miaomiao Huo ${ }^{1}$, Peng Wang ${ }^{2}$, Xianghe Liu ${ }^{1}$, Yu Wang ${ }^{1}$, Yinuo Li ${ }^{1}$, Zhixiang Zhou ${ }^{2}$, Ningzhi Xu ${ }^{1 凶}$ and
} Hongxia Zhu (iD ${ }^{1 凶}$

\begin{abstract}
Macrophages are among the most abundant immune cells in colorectal cancer (CRC). Re-educating tumor-associated macrophages (TAMs) to switch from protumoral to anti-tumoral activity is an attractive treatment strategy that warrants further investigation.

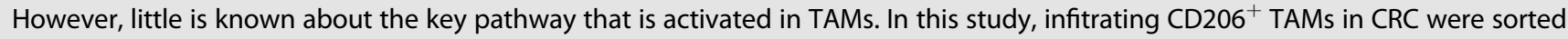
and subjected to RNA-seq analysis. Differentially expressed genes were found to be enriched in unfolded protein response/ endoplasmic reticulum stress response processes, and XBP1 splicing/activation was specifically observed in TAMs. XBP1 activation in TAMs promoted the growth and metastasis of CRC. Ablation of XBP1 inhibited the expression of the pro-tumor cytokine signature of TAMs, including IL-6, VEGFA, and IL-4. Simultaneously, XBP1 depletion could directly inhibit the expression of SIRPa and THBS1, thereby blocking "don't eat me" recognition signals and enhancing phagocytosis. Therapeutic XBP1 gene editing using AAV2-sgXBP1 enhanced the anti-tumor activity. Together, XBP1 activation in TAMs drives CRC progression by elevating pro-tumor cytokine expression and secretion, as well as inhibiting macrophage phagocytosis. Targeting XBP1 signaling in TAMs may be a potential strategy for CRC therapy.
\end{abstract}

Signal Transduction and Targeted Therapy (2021)6:357

; https://doi.org/10.1038/s41392-021-00761-7

\section{INTRODUCTION}

Colorectal cancer (CRC) is a fine example of a tumor tightly associated with its immune microenvironment. Not only colitis related CRCs, but also DNA damage or mutation-derived CRCs are enhanced by inflammation in the microenvironment. ${ }^{1}$ The infiltration of immune cells in CRCs contributes to tumor development, progression, and therapeutic response. ${ }^{2}$ Immunotherapy, which targets tumor-associated immune cells, is considered the new frontier of cancer treatment. Although many approaches, including PD-1/PD-L1 targeting and CTLA-4 targeting, have been tested during clinical trials of CRC patients, these have failed to achieve satisfactory beneficial effects. ${ }^{3}$ Therefore, the introduction of novel immunotherapies to clinical routines may be considered a priority.

Macrophages are among the most abundant immune cells observed in CRC, and those infiltrating in the tumor microenvironment are usually defined as tumor-associated macrophages (TAMs). ${ }^{4}$ Infiltration of the tumor front by $\mathrm{CD}^{+} 8^{+}$macrophages positively correlates with improved survival in colon cancer. ${ }^{5}$ However, another study showed that $\mathrm{CD}^{+} 8^{+}$TAM density is not a significant prognostic biomarker, whereas patients with high CD206 $^{+}$TAM density or high CD206/CD68 ratio showed significantly worse disease-free survival (DFS) and overall survival (OS) than those with a low density. ${ }^{6}$ Such conflicting data are attributed to the high plasticity of macrophages. ${ }^{7}$ Single cell RNAseq analyses identify two distinct subsets of TAMs in the tumor microenvironment of CRC. These two subsets show different functions, one subset expresses genes involved in phagocytosis and antigen presentation, whereas the other shows a more angiogenic signature. ${ }^{8}$ Therefore, depleting all TAMs may be problematic. In a tumor microenvironment, macrophages may switch from an anti-tumor phenotype to a pro-tumor one depending on the environment stimuli. ${ }^{9}$ Thus, re-educating TAMs to switch from protumoral to anti-tumoral activity is an attractive strategy that can be used to target macrophages and warrants further investigation. ${ }^{10,11}$

Activated unfolded protein response (UPR) is involved in most hallmarks of cancer. ${ }^{12}$ The IRE1a-XBP1 pathway is the most conserved process associated with UPR. When activated, IRE1a cleaves XBP1 mRNA, which induces the expression of activated $X B P 1$, leading to potent transcriptional activity. ${ }^{13}$ Recent studies have shown that the IRE1a-XBP1 pathway is involved in immune differentiation, activation, and cytokine expression in immune cells. ${ }^{14-16}$ XBP1 activation has been reported in dendritic cells and T cells in ovarian cancer microenvironments, and targeting XBP1 in dendritic cells or $T$ cells restored the anti-tumor immunity of these cells and thereby extended host survival. ${ }^{17,18}$ Endoplasmic reticulum (ER) stress also plays an important role in TAMs, by regulating the production of IL-6 ${ }^{19}$ and TNF-a. ${ }^{20}$ IL-4-induced macrophage polarization induces ER stress, wherein the inhibition of ER stress may block polarization. ${ }^{21,22}$ It is reported that IRE1 aXBP1 promotes macrophage activation to $M 1$ in white adipose tissue. ${ }^{23}$ The study shows that IRE1a downregulats Irf4 and KIf4 expression and suppresses $M 2$ polarization through a mechanism that requires its RNase activity but presumably not its Xbp1 mRNA splicing activity. Another study reported that an ER stress inhibitor

\footnotetext{
${ }^{1}$ State Key Laboratory of Molecular Oncology, National Cancer Center/National Clinical Research Center for Cancer/Cancer Hospital, Chinese Academy of Medical Sciences and

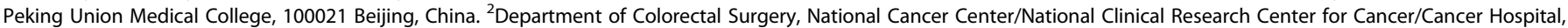
Chinese Academy of Medical Sciences and Peking Union Medical College, 100021 Beijing, China Correspondence: Ningzhi Xu (xuningzhi@cicams.ac.cn) or Hongxia Zhu (drhxzhu@cicams.ac.cn)
} 
inhibits lipopolysaccharides (LPS)-stimulated CD206 production in macrophages. ${ }^{24}$ In cancers, the pro-tumor functions of TAMs promote the expression of cell surface receptors, cytokines, chemokines, and enzymes, in addition to activating Treg cells or suppressing other effector cells. ${ }^{25}$ Inhibition of IRE1a-XBP1 in macrophages may attenuate CD86 and PD-L1 surface expression. ${ }^{26}$ Therefore, targeting XBP1 pathway of TAMs may be a novel strategy for CRC immunotherapy.

The current study evaluated the role of XBP1 in TAMs associated with colon cancer. XBP1 splicing was observed in TAMs of CRC patients and mouse models. XBP1 enhanced the pro-tumor function of TAMs. Deletion of XBP1 altered the cytokine a

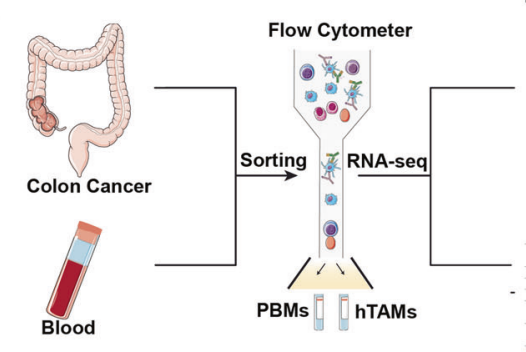

C

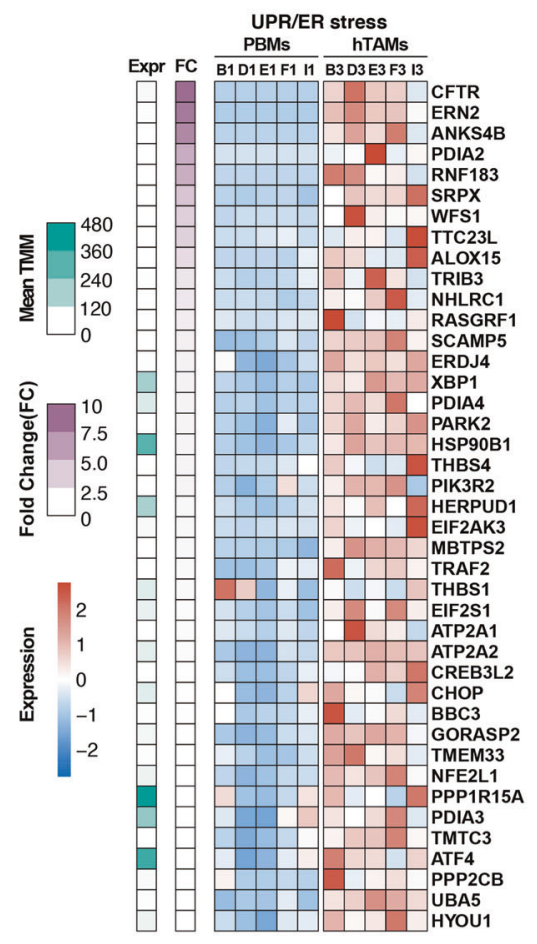

i

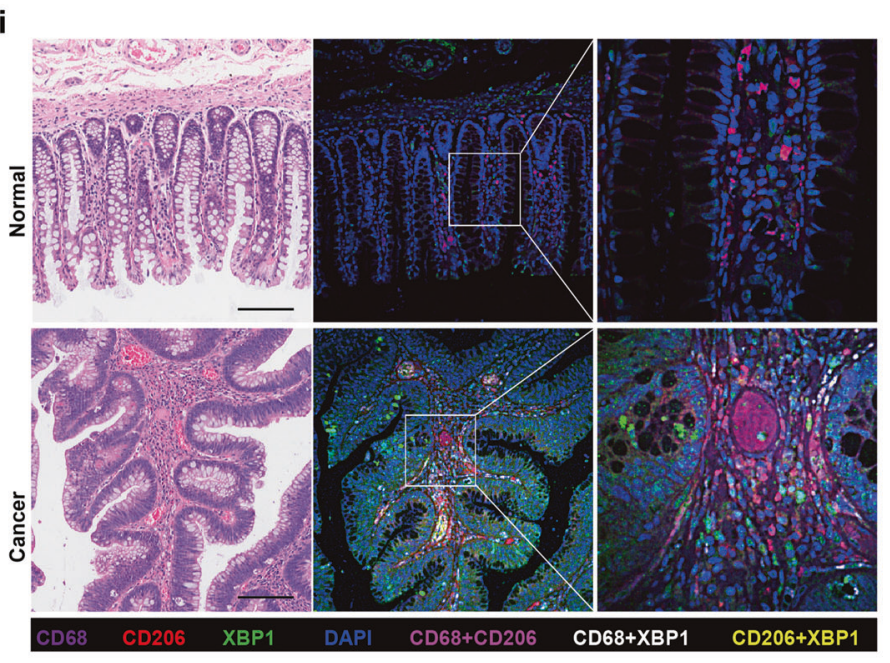

Differential Gene Expression

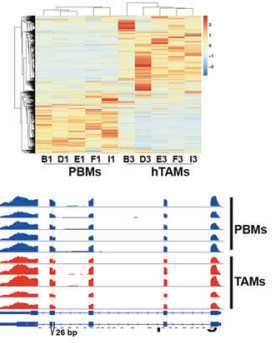

d

g
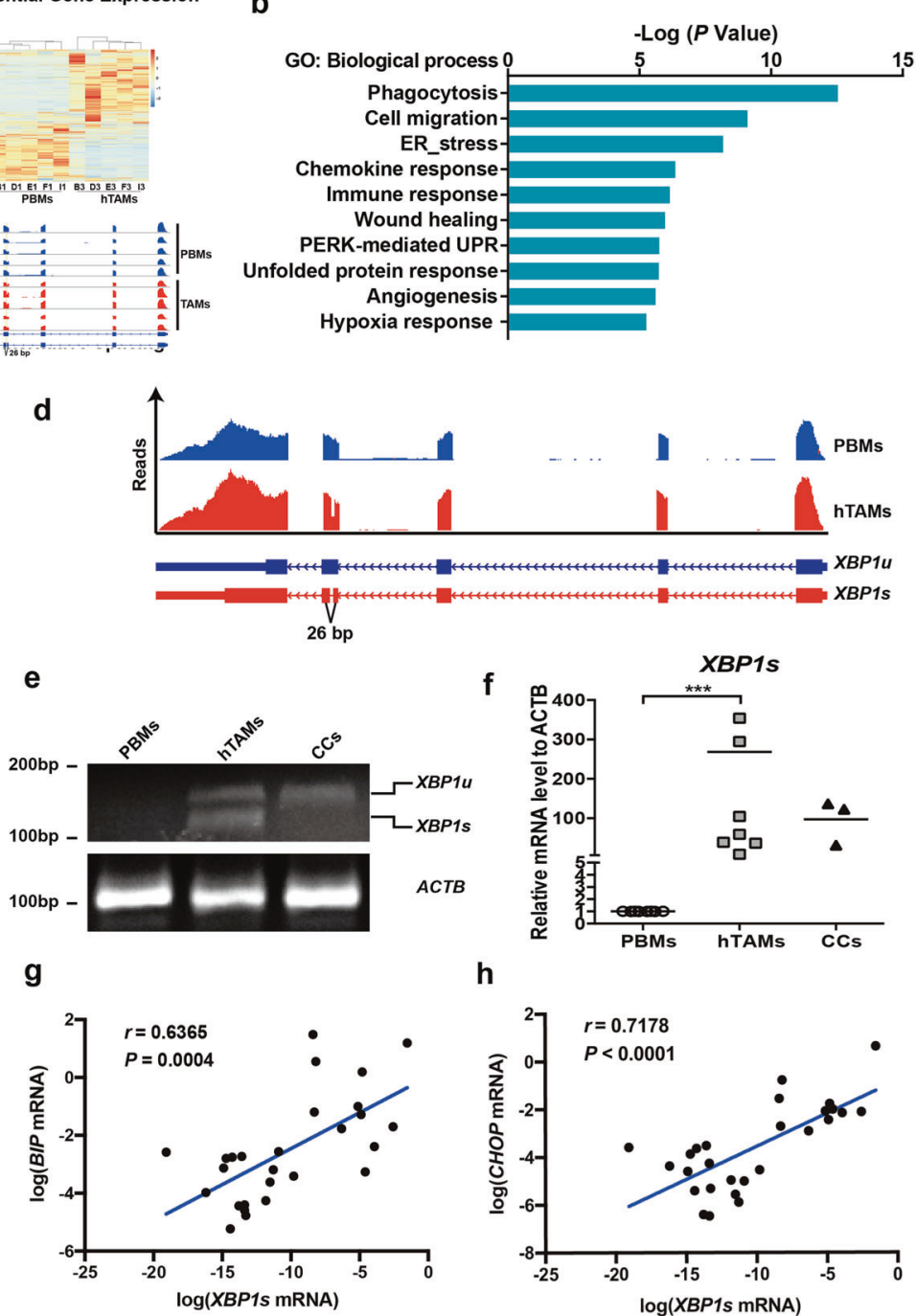

h
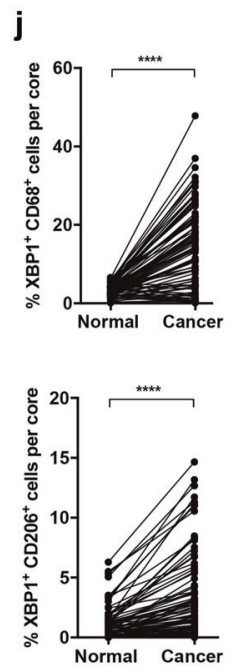

k
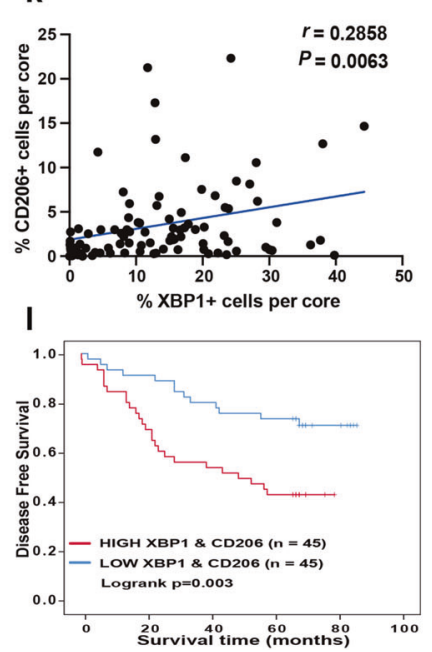
Fig. 1 UPR/ER-XBP1 activation in human TAMs infiltrate into CRC. a Schematic overview of the strategy for identification of UPR/ERXBP1 signaling pathways in hTAMs of CRC. $\mathbf{b}$ Gene Ontology (GO) term analysis of differentially regulated genes, as revealed using RNA-seq, in five paired hTAMs/PBMs samples. c Upregulation of genes involved in the UPR/ER stress response process. d XBP1 splicing in hTAMs confirmed by RNA-seq alternative mRNA splicing analysis. XBP1u, unspliced form; XBP1s, spliced form. e Detection of XBP1 splicing using conventional RT-PCR and agarose gel electrophoresis. $\mathbf{f}$ Expression of XBP1s in PBMs, hTAMs and cancer cells evaluated by RT-qPCR. Data were normalized to endogenous levels of $A C T B$. ${ }^{* * *} P<0.001$; ANOVA test. $\mathbf{g}, \mathbf{h}$ Expression of BIP and CHOP versus XBP1s in all hTAM samples from CRC patients $(n=27)$. $\mathbf{r}$ Spearman's rank correlation test. i CD206, CD68, and XBP1 immunofluorescence stains in human CRCs and adjacent normal tissues. Scale bar: $100 \mu \mathrm{m}$. $\mathbf{j ~ X B P} 1^{+} \mathrm{CD} 68^{+}$cells (upper panel), and XBP1 ${ }^{+} \mathrm{CD} 206^{+}$cells (lower panel) among the total number of cells in each individual core from human CRCs and adjacent normal tissues, as determined by PerkingElmer inFormTM system. ${ }^{* * * *} P<0.0001$; paired $t$-test. k Correlation of percentage of XBP1 ${ }^{+}$cells with CD206 ${ }^{+}$in CRC patients. $r$, Spearman's rank correlation test. I Kaplan-Meier survival curves for 90 CRC patients with or without high $\mathrm{XBP} 1^{+} \mathrm{CD} 206^{+}$cells

expression signature and promoted macrophage phagocytosis of tumor cells by disrupting self-recognition. Our results suggested that XBP1 in TAMs had potential as a novel therapeutic target in human colon cancer.

\section{RESULTS}

TAMs infiltrating into CRC exhibit XBP1 splicing and activation According to the Cancer Genome Atlas (TCGA) RNA-seq data, macrophage is one of the most abundant cell types infiltrating in CRC (Supplementary Fig. S1a). TAMs accumulated in CRCs are associated with tumor progression and the efficacy of therapeutics. ${ }^{5,6}$ To investigate the mechanism by which TAMs interact with the tumor microenvironment, $\mathrm{CD} 14^{+} \mathrm{CD} 11 \mathrm{~b}^{+}$peripheral blood cells and $\mathrm{CD}_{14}{ }^{+} \mathrm{CD} 11 \mathrm{~b}^{+} \mathrm{CD} 206^{+}$intra-tumoral human CRCassociated macrophages (hTAMs) were isolated from patients with CRC and subjected to RNA-seq analysis (Fig. 1a). Although CD206 ${ }^{+}$ macrophages accounted for the majority of $\mathrm{CD} 14^{+} \mathrm{CD} 11 \mathrm{~b}^{+}$cells isolated from the tumor, very few were observed in peripheral blood monocytes (PBMs) (Supplementary Fig. S1b, c), indicating that $\mathrm{CD}_{206}{ }^{+}$hTAMs accumulated in the CRC tumor microenvironment. RNA-seq results showed that differentially expressed genes were enriched in ER stress and UPR process (Fig. 1b). Many known UPR/ER stress genes were upregulated in hTAMs compared with PBMs (Fig. 1c). Moreover, XBP1 splicing was detected in hTAMs from all five samples, but not in PBMs (Fig. 1d). Reverse transcriptase PCR (RT-PCR) results indicated that XBP1 mRNA splicing in TAMs was increased compared with that in PBMs and cancer cells (Fig. 1e). Quantitative analyses consistently confirmed that the expression level of spliced XBP1 mRNA in hTAMs was increased in hTAMs compared with that in control PBMs and cancer cells (Fig. 1f). Furthermore, expression levels of the ER stress markers, BIP and CHOP, were positively correlated with spliced $X B P 1$ in the hTAMs of CRC samples (Fig. 1g, h). Next, we included the multilabel immunofluorescence (MIF) of XBP1, CD206 and CD68 in CRC tissue array (Supplementary Fig. S2a and Fig. 1i). Cancerous lesions had more infiltration of $\mathrm{XBP} 1^{+} \mathrm{CD} 68^{+}$cells (white) compared to normal tissue $(14.11 \pm 1.10$ vs. $3.40 \pm 0.18 ; P<$ $0.0001), \mathrm{XBP} 1^{+} \mathrm{CD} 206^{+}$macrophages displayed the similar pattern (3.26 \pm 0.37 vs. $1.07 \pm 0.15 ; P<0.0001$ ) (Fig. 1j). Eexpression of XBP1 and CD206 in TAMs was correlated with poor survival of CRC patients (Supplementary Fig. S2b, c). Furthermore, the infiltration of $\mathrm{XBP} 1^{+} \mathrm{TAMs}$, especially $\mathrm{XBP} 1^{+} \mathrm{CD} 206^{+}$(representing the XBP1 activation in TAMs), in tumors was associated with shorter DFS in $\mathrm{CRC}$ patients, but not $\mathrm{XBP} 1^{+} \mathrm{EPCAM}^{+}$(representing the XBP1 activation in cancer cells) (Fig. $1 \mathrm{k}, \mathrm{I}$ and Supplementary Fig. S2d, e). Moreover, the high frequency of XBP $1^{+} \mathrm{CD} 206^{+}$TAMs (representing the XBP1 activation in TAMs) were significantly correlated with TNM stage (Fisher Exact test, $P=0.0314$ ) (Supplementary Fig. S2f). XBP1 expression was highly correlated with the TAM markers, CD206 and CD163, which was also confirmed using a public GEO databases (GSE14333) (Supplementary Fig. S2i). Expression of XBP1 and CD206 was strongly associated with shorter DFS in GEO databases GSE38832 (Supplementary Fig. S2g, h) and GSE14333 (Supplementary Fig. S2j, k).
Furthermore, an AOM-DSS-induced CAC model was established (Supplementary Fig. S3a). Following three cycles of induction, tumors became visible in the colon, upon which the mice were sacrified (Supplementary Fig. S3b, c). Immunofluorescence staining demonstrated that XBP1 was expressed in macrophages infiltrating in cancerous lesions $\left(\mathrm{XBP} 1^{+} \mathrm{F} 4 / 80^{+}\right.$and $\mathrm{XBP} 1^{+} \mathrm{CD} 206^{+}$ cells) but not in $\mathrm{F} 4 / 80^{+}$macrophages infiltrating in normal tissues (Fig. 2a). Mouse TAMs (mTAMs) from tumor colorectal lesions and spleen monocytes were sorted (Supplementary Fig. S3d) and applied to RNA-seq analysis. Differentially expressed genes were enriched in stress-related processes (Fig. 2b). RT-PCR and agarose gel electrophoresis indicated that although mTAMs sorted from tumors of mouse AOM-DSS model contained spliced Xbp1, no splicing was detected in macrophages sorted from the spleen (Fig. 2c). Xbp1s expression was significantly higher in mTAMs than that in control spleen monocytes (Fig. 2d). ER stress markers, Bip and Chop, were also upregulated in mTAMs (Fig. 2e, f). The above results indicated that although macrophages derived from tumor tissues showed XBP1 activation, those derived from normal tissues did not. Taken together, TAMs infiltrating in CRC exhibit $\mathrm{XBP} 1$ splicing and activation.

XBP1 activation in TAMs promotes the growth and metastasis of CRC

We next investigated the contribution of XBP1 in TAMs associated with tumor growth and metastasis. TAMs enhanced tumor growth of the subcutaneously injected luciferase tumor cells (CT26luciferase) in NOD/SCID mice (Fig. 3a-c), while knockout of XBP1 in miTAMs inhibited tumor growth (Fig. $3 d-f$ and Supplementary Fig. S4a-c). Inhibition of XBP1 splicing by IRE1a inhibitor $4 \mu 8 \mathrm{c}$ also diminished tumor-promoting function of TAMs (Ana-1 conditioned medium [CM]) (Supplementary Fig. S4d-f). Next, we determined whether XBP1 activation in TAMs promoted metastasis of CRC cells. Metastasis of CT26 cells was analyzed using the Boyden chamber assay (Supplementary Fig. S4g). While TAMs (Ana-1 CM) enhanced the migration of CT-26 cells, knocking out of XBP1 in TAMs inhibited CT26 cell migration (Supplementary Fig. $\mathrm{S} 4 \mathrm{~h}, \mathrm{i}) . \mathrm{NOD} / \mathrm{SCID}$ mice were pretreated with clodronate liposomes for two weeks to eliminate intrinsic macrophages. CT26 cells mixed with Con TAMs, Xbp1s TAMs, and sgCon TAMs, and sgXbp1 TAMs, were injected orthotopically into the wall of the cecum. After four weeks, incidence of orthotopic CRC tumor formation and HE staining of liver metastasis analysis were observed (Fig. 3g, h). Compared with control group, mice injected with miTAMs XBP1s-overexpressing miTAMs showed an increase in orthotopic tumor formation (4/5 vs. $5 / 5$ ) and liver metastasis (3/5 vs. $5 / 5$ ). Conversely, mice harboring TAMs lacking XBP1 showed reduced orthotopic tumor formation (5/5 vs. $3 / 5)$ and liver metastasis (4/5 vs. 1/5) compared with control mice. Quantification of the orthotopic CRC tumor weight and the clone number for liver metastasis confirmed that co-injection with XBP1s-overexpression TAMs could lead to a significant increase in the tumor burden of CRC orthotopic tumor burden and liver metastasis, and vice versa (Fig. $3 \mathrm{~g}-\mathrm{k}$ ). Furthermore, spleen injection model of liver metastasis also indicated that mice injected with XBP1s-overexpressing 
Zhao et al.
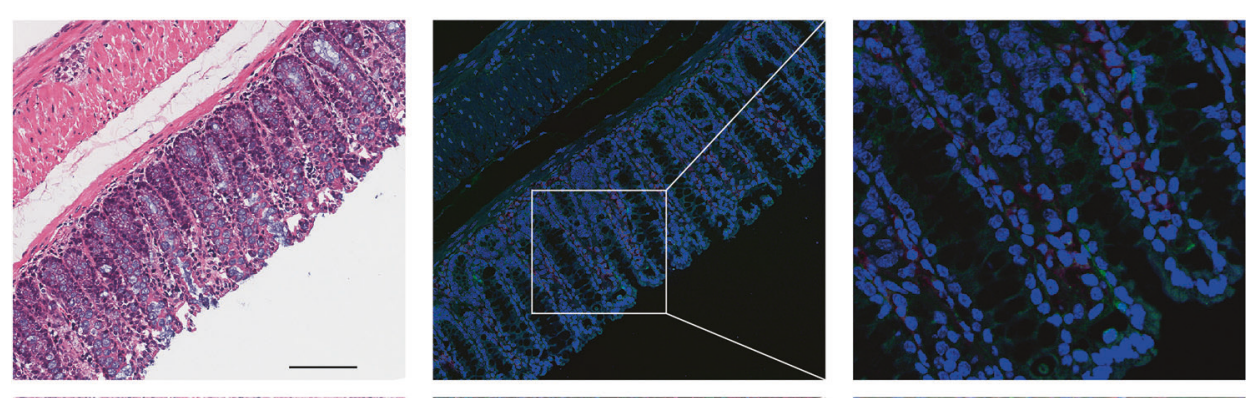

은
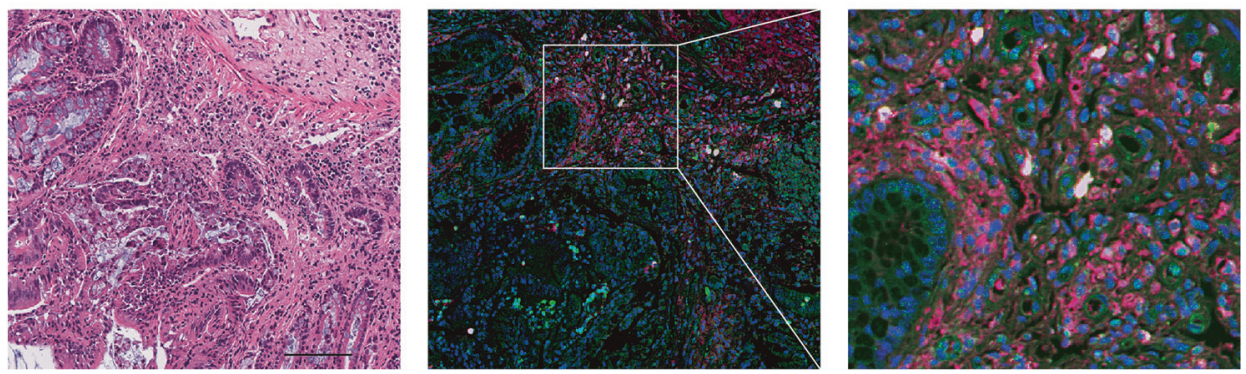

F4/80 CD206 XBP1 DAPI F4/80+CD206 F4/80+XBP1 CD206+XBP1

b

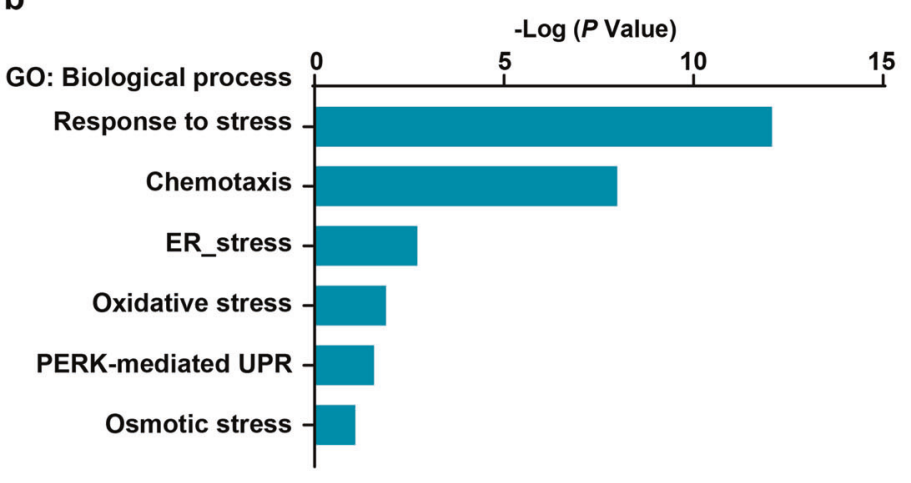

C

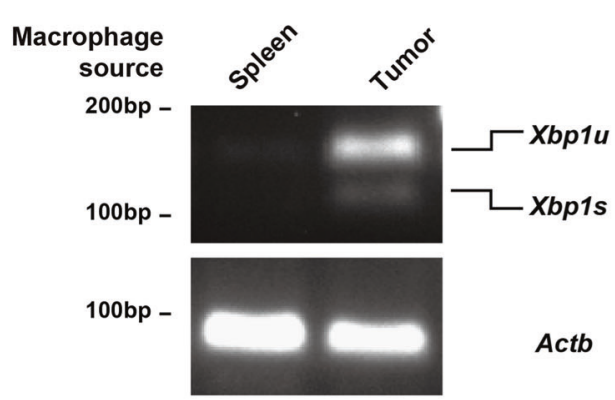

d

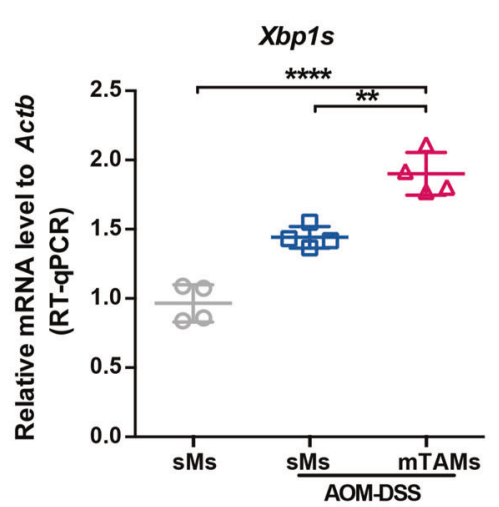

e

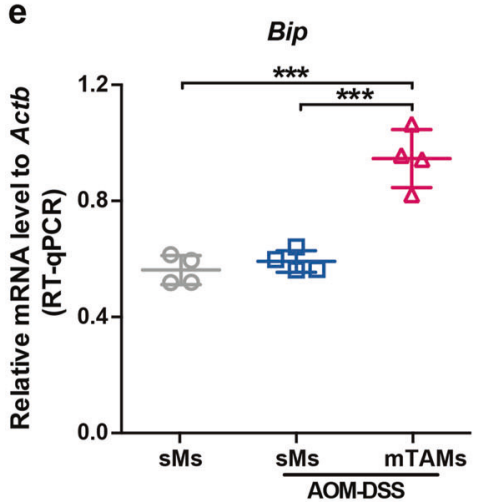

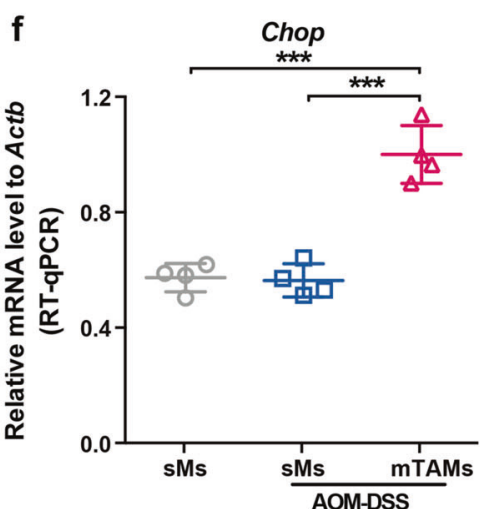

Fig. 2 XBP1 activation in AOM-DSS-induced colorectal cancer-associated macrophages. a F4/80, CD206, and XBP1 immunofluorescence in colon sections from healthy and AOM-DSS mice. Scale bar: $100 \mu \mathrm{m}$. b Gene Ontology (GO) term analysis of differentially regulated genes, as revealed using RNA-seq, in four paired mTAMs and spleen macrophages (sMs). c Xbp1 splicing was evaluated using conventional RT-PCR and agarose gel electrophoresis. $\mathbf{d}$ Expression of XBP1s in spleen macrophages (sMs) and mTAMs evaluated via RT-qPCR. Data were normalized to endogenous levels of $A C T B$. e, $\mathbf{f}$ Expression of the UPR/ER stress response transcripts Bip and Chop determined by RT-qPCR. Data normalized to endogenous levels of $A C T B$. ( $n=4$ mice per group); ${ }^{* *} P<0.01,{ }^{* * *} P<0.001,{ }^{* * * *} P<0.0001$; ANOVA test 
miTAMs showed an increase in liver metastasis, compared with control mice $(25.92 \pm 2.41$ vs. $9.76 \pm 1.85 ; P<0.01)$, whereas mice harboring TAMs lacking XBP1 showed reduced liver metastasis compared with control mice $(1.13 \pm 0.51$ vs $11.47 \pm 1.13 ; P<0.001)$; (Supplementary Fig. S4j, k). Similarly, CT-26 injected via the tail vein promoted the formation of metastatic nodules in the lung.
Histological staining confirmed that the cells were cancerous (Fig 3l). Mice injected with XBP1s-overexpressing miTAMs displayed increased number of lung nodules compared with those in control mice $(33.75 \pm 2.14$ vs $15.00 \pm 1.78 ; P<0.001)$. In contrast, mice lacking XBP1 in miTAMs showed reduced number of lung nodules compared with control mice $(9.75 \pm 1.55$ vs $22.25 \pm 1.49 ; P<0.01)$; a

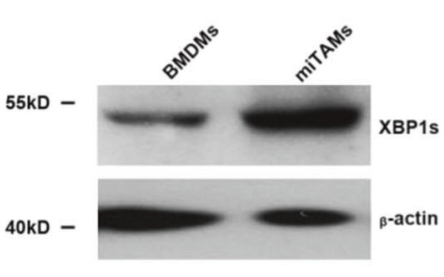

d

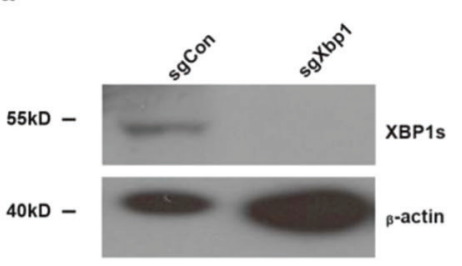

b

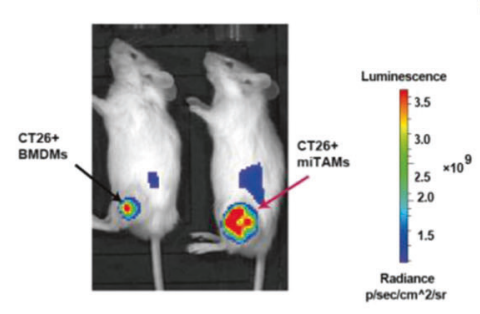

C
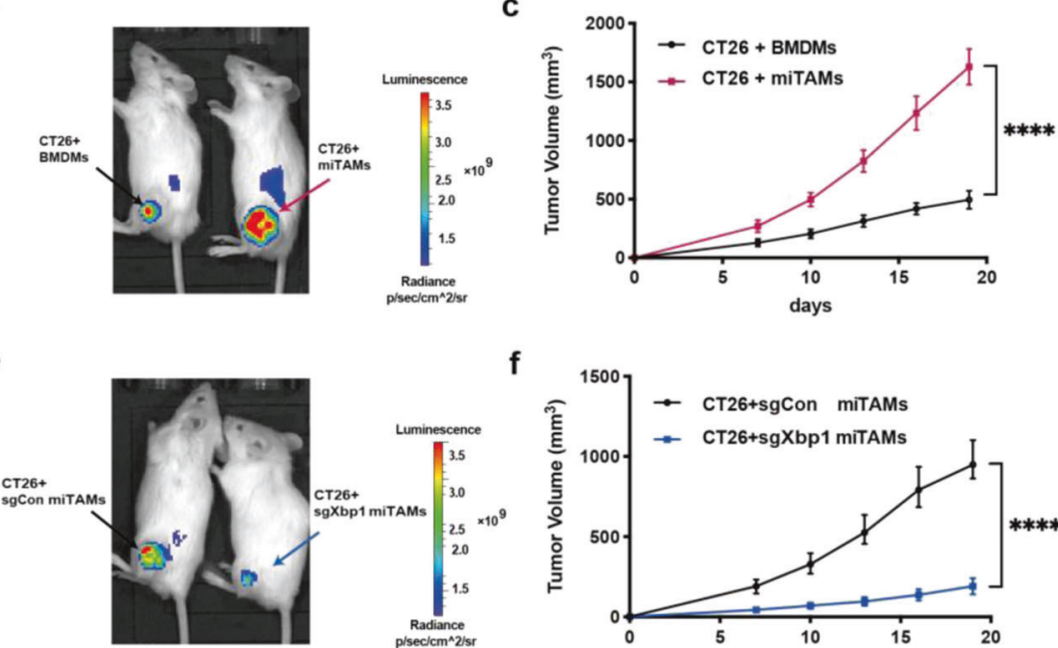

$f$

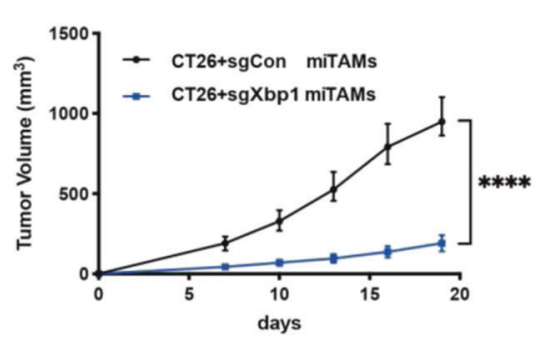

g
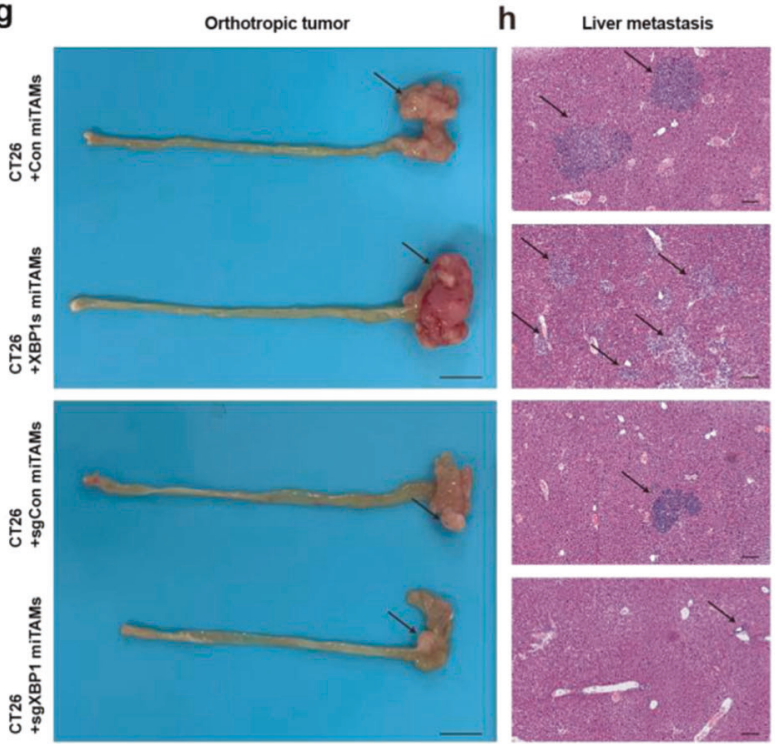

\section{I}

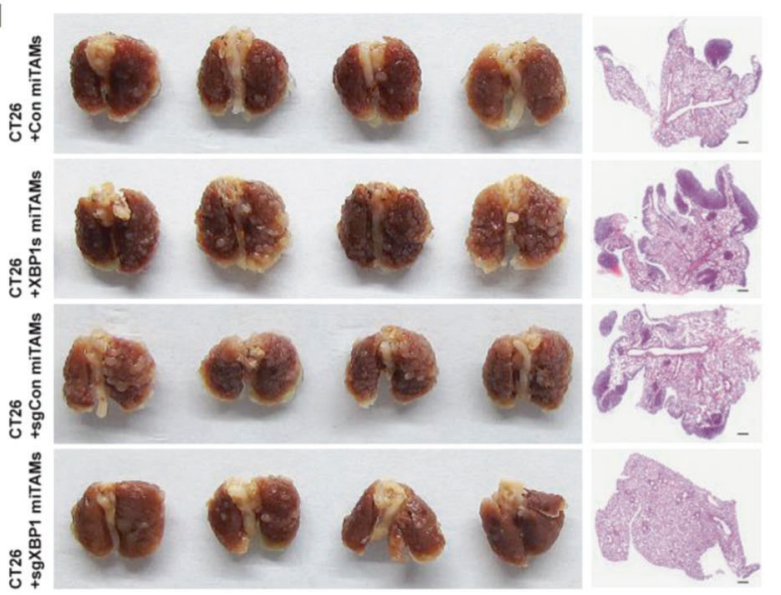

i

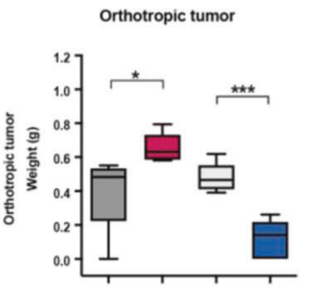

j

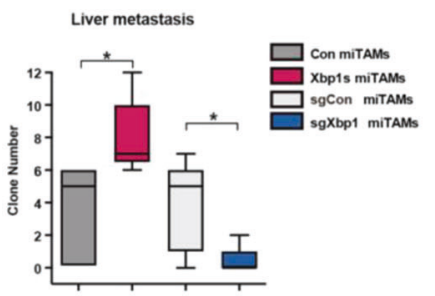

$\mathbf{k}$

\begin{tabular}{lcc}
\multicolumn{2}{l}{ Incidence of tumor formation } \\
\hline Groups & Orthotropic tumor & Liver metastasis \\
\hline Con miTAMs & $4 / 5$ & $3 / 5$ \\
XBP1s miTAMs & $5 / 5$ & $5 / 5$ \\
sgCon miTAMs & $5 / 5$ & $4 / 5$ \\
SgXBP1 miTAMs & $3 / 5$ & $1 / 5$ \\
\hline
\end{tabular}

m Lung metastasis

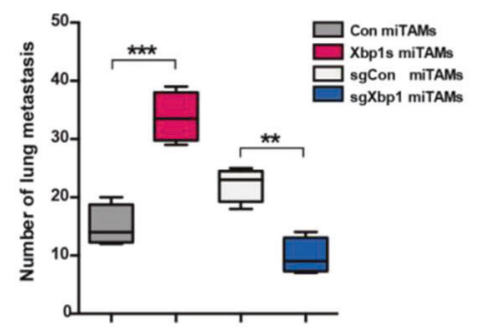


Fig. 3 Effect of XBP1 activation on the pro-tumor function of TAMs. a Western blot analysis of XBP1s expression in indicated macrophages. b Representative micrograph showing tumor formation in NOD/SCID mice injected subcutaneously (s.c.) with luciferase tumor cells (CT26luciferase) and two groups of macrophages: CT26 + BMDMs (black arrows) and CT26 + miTAMs (magenta arrows). c Growth curves of the two groups in b. d Western blot analysis of XBP1 expression in sgCon or sgXbp1 TAMs. e Representative photograph showing tumor formation in NOD/SCID mice injected s.c. with luciferase tumor cells (CT26-luciferase) and two groups of miTAMs: CT26 + sgCon miTAMs (black arrows); and CT26 + sgXbp1 miTAMs (blue arrows). f Growth curves of the two groups in e. ${ }^{* * * P} P<0.0001$; Repeated measurement and analysis. g CT26 cells, mixed with: Con TAMs; Xbp1s TAMs; sgCon TAMs; and sgXbp1 TAMs, were orthotopically injected into the wall of the cecum ( $n=5$ mice per group). Macroscopic appearance of the CRC orthotopic tumors with each indicated treatment. Black arrows indicate macroscopic polyps. Scale bar, $1 \mathrm{~cm}$. h Representative HE staining of liver metastasis in mice xenografted of the four groups in $\mathbf{g}$. Scale bar, $100 \mu \mathrm{m} . \mathbf{i}, \mathbf{j}$ Statistical analysis of the orthotopic CRC tumor weight (i) and the clone number of liver metastasis (j). ${ }^{*} P<0.05,{ }^{* * *} P<0.001 ; t$-test. k Incidence of orthotopic CRC tumor formation and liver metastasis analysis. I Representative images of CT26 pulmonary metastases induced by tail vein injection in NOD/SCID. CT26 cells were mixed with: Con miTAMs; Xbp1s miTAMs; sgCon miTAMs; and sgXbp1 miTAMs. HE staining demonstrating the histology of tumors formed in the lungs; scale bar, $500 \mu \mathrm{m}$. $\mathbf{m}$ Pulmonary metastatic nodule numbers in i. $(n=4 \mathrm{mice}$ per group); ${ }^{* *} P<0.01,{ }^{* * *} P<0.001 ; t$-test

(Fig. 3m). Taken together, XBP1 activation in TAMs was necessary for the growth and metastasis of CRC in mouse models.

\section{XBP1 activation regulates the cytokine expression signature of TAMs}

Cytokines released by macrophages influence cancer cell proliferation and migration. ${ }^{27,28}$ To determine whether XBP1 promotes tumor growth by regulating cytokine expression in TAMs, cell supernatants of TAMs and XBP1-knockout TAMs were applied to cytokine array assay. The concentrations of IL-4, IL-6, and VEGFA, decreased significantly in XBP1 knocked-out miTAMs, whereas TNFa increased (Fig. 4a). RT-qPCR indicated that expression of II-4, II-6, Mmp2, Vegfa, II-33, Pdgfa, and Tgfb1 was upregulated in miTAMs compared with that in original bone marrow-derived macrophages (BMDMs), but decreased when XBP1 was knocked out. In contrast, Tnfa expression, which was decreased in TAMs, was found to be increased in sgXBP1 miTAMs (Fig. 4b). RT-qPCR showed similar results in tumor condition medium induced human macrophage THP-1 cells (Fig. 4c). ELISA results indicated that the concentrations of VEGFA and IL- 6 were upregulated in TAMs (THP-1 cell induced by HCT116 CM; THP-1 CM) compared with those in THP-1 cells, but downregulated upon XBP1 knockout (Supplementary Fig. S5a, b). Meanwhile, track view of ChIP-seq density profiles in the GSE86048 database indicated that XBP1 knockdown reduced the binding efficacy of Vegfa, II-4 and II-6 sequences (Supplementary Fig. S5c). Nuclear extracts from control TAMs or sgXbp1 TAMs were subjected to ChIP using antiXBP1 antibodies. The promoter regions of Vegfa, II-4 and II-6 were enriched in the immunoprecipitates with anti-XBP1 antibody, but knock-down of XBP1 decreased the binding of these promoters (Fig. 4d). Moreover, RT-qPCR showed that the expression levels of VEGFA, IL-4 and IL-6 were positively correlated with that of XBP1 in hTAMs (M2-like) derived from CRC patients (Fig. 4e). A similar relationship was also found in the tumor samples of CRC patients (GSE38832), as shown in Supplementary Fig S5d. High expression of VEGFA, IL-4 and IL- 6 was associated with shorter DFS in CRC patients (GSE38832) (Fig. 4f). These results demonstrate that XBP1 directly regulates the transcription signature of cytokines in macrophages and thereby enhances cancer progression.

XBP1 inhibition promotes macrophage phagocytosis by disturbing the self-recognition

In addition to releasing cytokines that modulate tumor progression, TAMs clear tumor cells directly via phagocytosis. To evaluate whether XBP1 affects phagocytosis, we co-cultured RFP-labeled DLD1 with hiTAMs (Peripheral blood mononuclear cell (PBMC)-derived macrophages), and cocultured RFP-labeled CT26 with miTAMs (BMDMs). Following $8 \mathrm{~h}$ of co-culture, compared with the sgCon TAMs, the RFPlabeled cancer cells (red) were efficiently phagocytosed by sgXBP1 hiTAMs or sgXbp1 miTAMs, indicating that XBP1 knockout increased the phagocytic capacity of TAMs remarkably (Fig. 5a-c). The phagocytic activity of macrophages is regulated by activating ("eat") as well as inhibitory ("do not eat") signals. CD47, a widely expressed transmembrane glycoprotein, suppresses phagocytosis by binding to SIRPa and THBS1 on the surface of macrophages. ${ }^{29,30}$ SIRPa and THBS1 levels were upregulated in miTAMs compared with those in BMDMs, but inhibition of XBP1 significantly reduced the expression levels (Fig. 5d). Similar results were observed in THP-1 cells (Supplementary Fig. S6a). The expression pattern of SIRPa and THBS1 were similar to that of ERDJ4, a target of XBP1. RT-qPCR showed that the expression levels of SIRPa and THBS1 were positively correlated with that of XBP1 in hTAMs derived from CRC patients (Fig. 5e). A similar relationship was also found in the tumor samples of CRC patients (GSE68468, GSE38832), as shown in Fig. $5 f$ and Supplementary Fig. S6c. THBS1 and SIRPa expression was correlated with poor prognoses for CRC patients (Supplementary Fig. S6d). Then, we sought to investigate whether SIRPa and THBS1 were transcriptional targets of XBP1. Track view of ChIP-seq density profile indicated that Erdj4, Thbs1, and Sirpa (GSE86048) in the livers of XBP1knockdown mice showed reduced binding efficacy (Fig. 5g). ChIP experiments showed that the promoter regions of Erdj4, Thbs1, and Sirpa were enriched in the immunoprecipitates with anti-XBP1 antibody but knockout of Xbp1 led to decreased binding of these promoters in miTAMs (Fig. $5 \mathrm{~h}$ ). Therefore, XBP1 directly regulate the transcription of THBS1 and SIRPa. XBP1 inhibition promoted macrophage phagocytosis of tumor cells by disturbing the selfrecognition of TAMs.

\section{Therapeutic knockout of XBP1 enhances anti-tumor activity of TAMs}

Then, the role of XBP1 in TAMs in tumor progression was evaluated via AOM-DSS model. Clodronate liposome (clodrolip) reduces the number of macrophages infiltrating tumors, limits tumor metastasis in lung cancer. ${ }^{31}$ Therefore, we depleted all macrophages using clodrolip injections in AOM-DSS mouse model (Fig. 6a), but there was no significant difference between the polyp numbers of treatment and control groups (Fig. 6b, c). Phagocytosis checkpoints are now considered as promising targets for cancer immunotherapy. ${ }^{32}$ CD47/SIRPa axis is one of the most important pathways, which enables tumor cells to evade phagocytosis by macrophages. Blockage of the CD47/SIRPa stimulates phagocytosis of cancer cells by macrophages in vitro and inhibits tumor growth in vivo. ${ }^{33}$ Therefore, we used therapeutic SIRPa antibodies to treat AOM-DSS-induced CRC. Tumor numbers were significantly reduced by SIRPa antibody treatment (Fig. 6d-f). Previous results showed that targeting XBP1 inhibited the growth and metastasis of CRC in mouse models. We evaluated the therapeutic effect of XBP1 silencing on AOM-DSS model. To selectively target XBP1 in macrophages, sgRNA-Xbp1 adeno-associated virus was produced as described in methods. Lyz2-Cre-LSL-Cas9 mice were inoculated with AAV2- sgXbp1 one week following the second DSS cycle, and euthanized five weeks later (Fig. $6 \mathrm{~g}$ ). To test the efficacy and specificity of AAV2-sgXbp1 on the expression of XBP1 in mTAMs, we determined the MIF of 
a

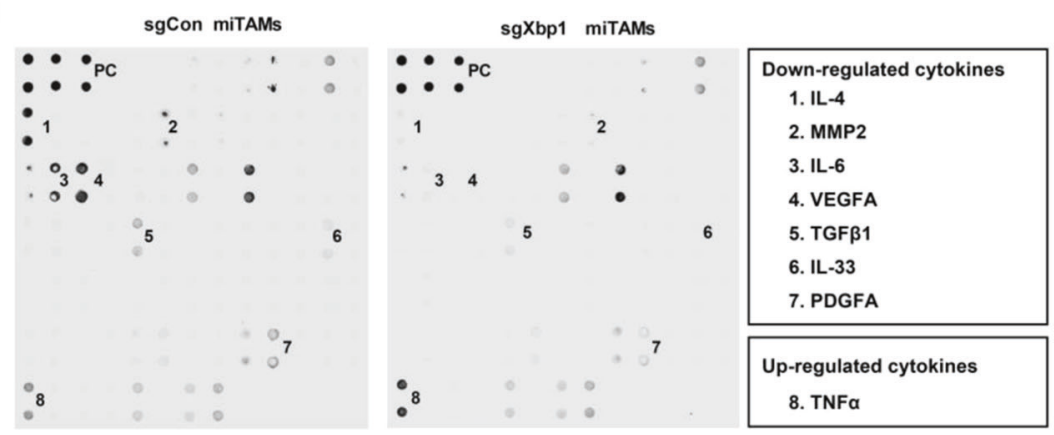

c

d

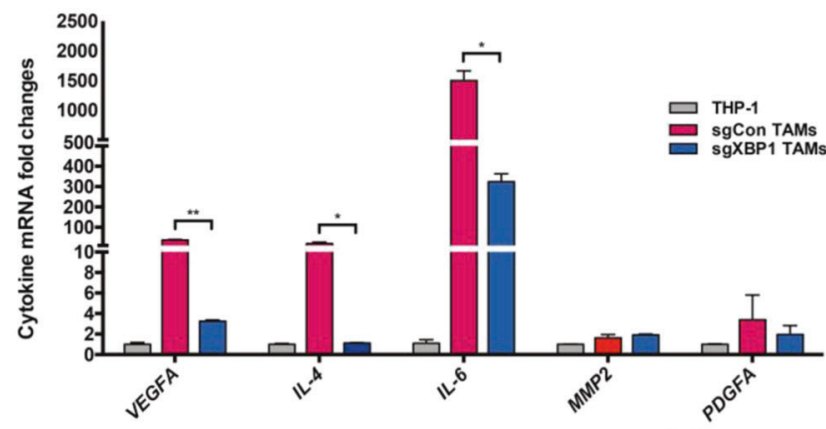

b

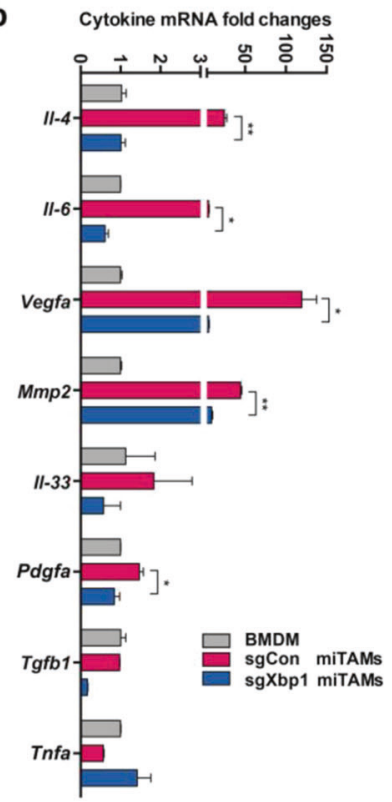

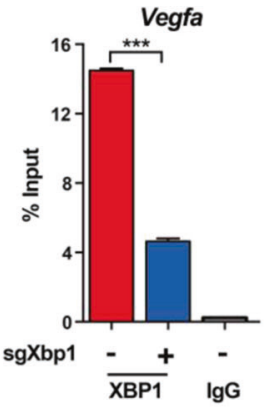
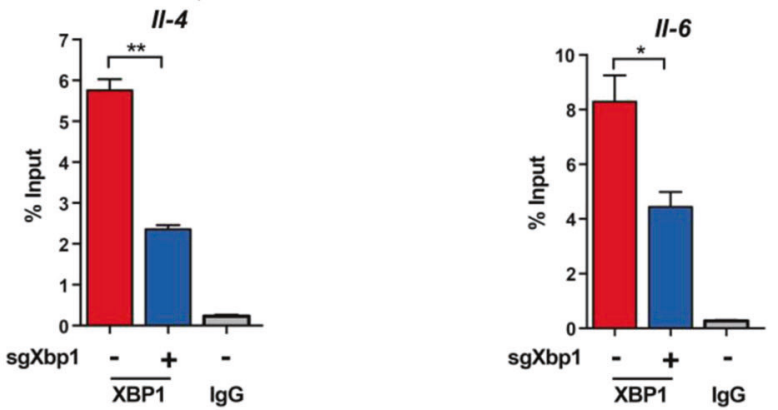

e
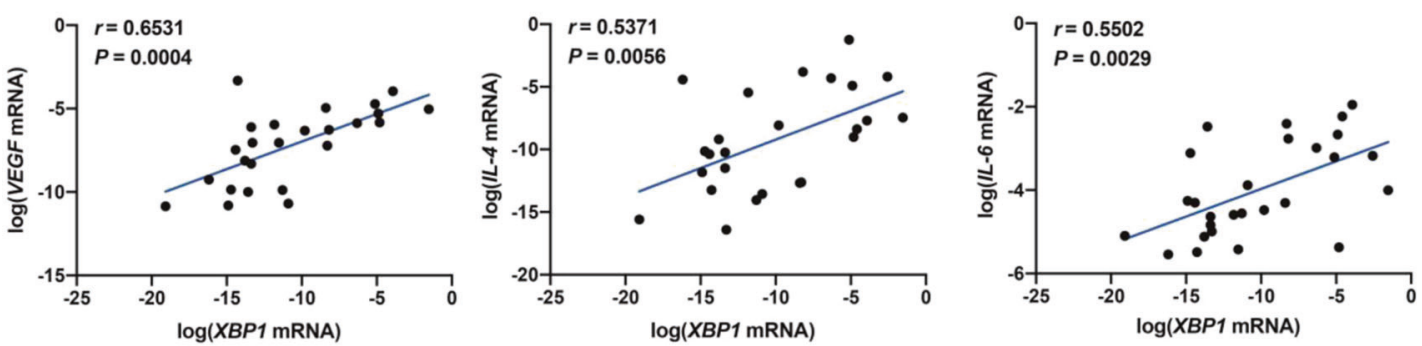

f

GSE38832

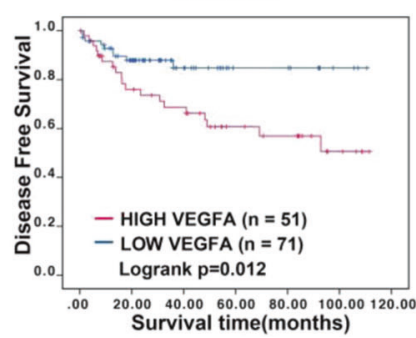

GSE38832

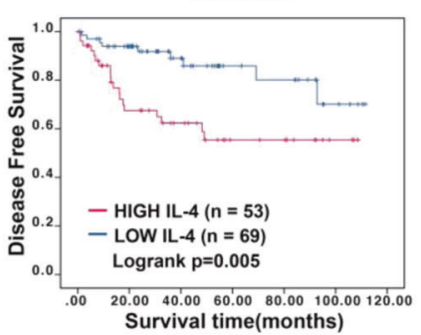

GSE38832

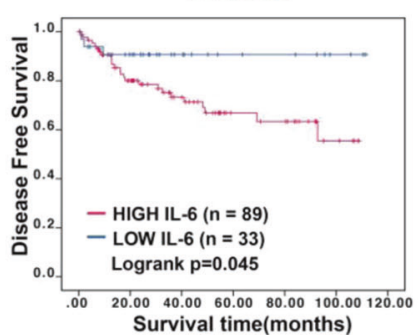

Fig. 4 Cytokines production induced by XBP1 activation in TAMs. a Representative cytokine arrays for sgCon miTAMs and paired sgXbp1 miTAMs supernatants. b Relative mRNA levels of cytokines in BMDMs, sgCon miTAMs, and paired sgXbp1 miTAMs validated by RT-qPCR. c Expression of cytokines in human TAMs. Human macrophages from THP-1 cells were induced to TAMs via incubation in condition medium of HCT116 cells, and relative mRNA levels of cytokines in THP-1, sgCon or sgXBP1 TAMs were validated by RT-qPCR. d ChIP-qPCR experiments measuring XBP1 binding on Vegfa, II-4, and II-6 segments. Bars represent mean \pm SD of three experimental replicates. ${ }^{*} P<0.05$, ${ }^{* *} P<0.01$, ${ }^{* * *} P<0.001$; $t$-test. e Expression of VEGFA, IL-4, and IL-6 versus XBP1s in all hTAM samples from CRC patients $(n=27) . r$, Spearman's rank correlation test. $\mathbf{f}$ Kaplan-Meier survival curves for CRC patients with or without high expression levels of VEGFA, IL-4 and IL-6 in the GEO online database (GSE38832). The optimal survival cut point was determined via X-Tile statistical software 
F4/80, CD206, and XBP1 in colon sections from AAV2-sgCon and AAV2-sgXbp1 AOM-DSS mice. We detected the expression of $\mathrm{XBP} 1$ in colorectal cancer tissue from mice model treateds with AAV2-sgXbp1, as shown in Supplementary Fig. S7a-d; the expression of XBP1 decreased in $\mathrm{F} 4 / 80^{+}$macrophages but not in epithelium cells. The result indicated that the anti-tumor function was a result of knocking out XBP1 from TAMs but not from tumor cells. Conditional deletion of XBP1 in TAMs significantly inhibited tumor formation in AOM-DSS mouse model
(Fig. $6 \mathrm{~h}, \mathrm{i}$ ). To test the therapeutic effects of targeting XBP1 in macrophages in human models, we employed CRC PDXs to for coinjection with hiTAMs (Fig. 6j, k). Knockout of XBP1 in hiTAMs inhibited PDX tumor growth. Further, mTAMs isolated from mice treated with AAV2-sgXbp1 showed reduced expression and secretion of IL-4, IL-6 and VEGFA (Supplementary Fig. S7e, f). The expression of recognition signals (Sipra, Thbs1, Pd-I1, Pd-1, Siglec-10, and H2-k1), especially Sipra, also decreased in AAV2sgXbp1 mTAMs (Supplementary Fig. S7g). These data indicated a

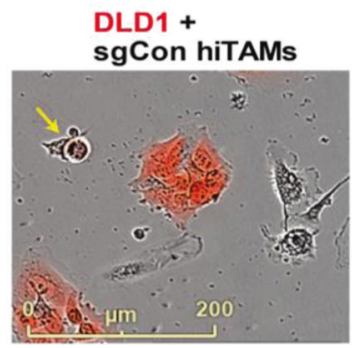

C

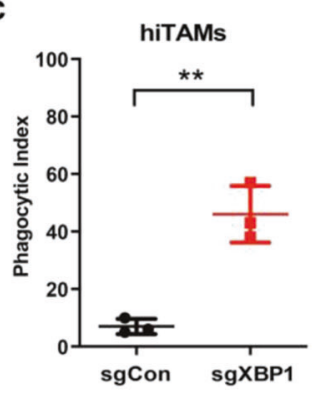

e

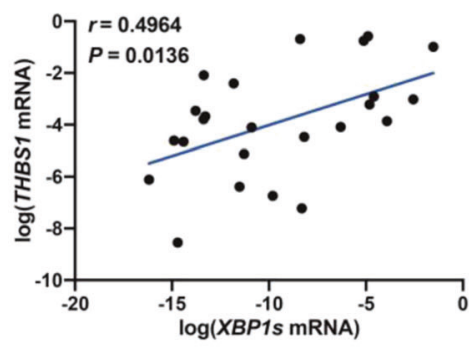

f

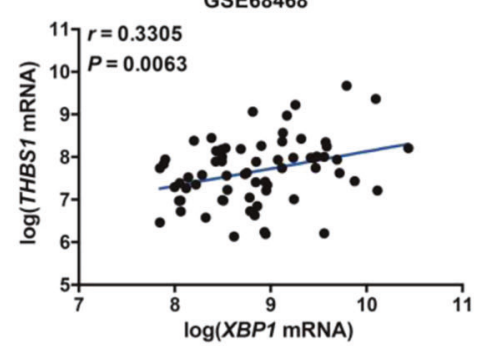

b

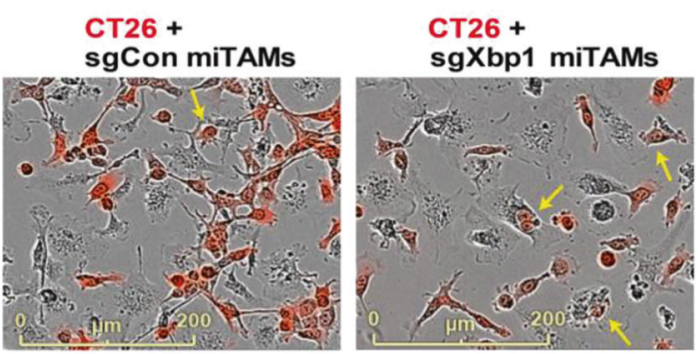

d

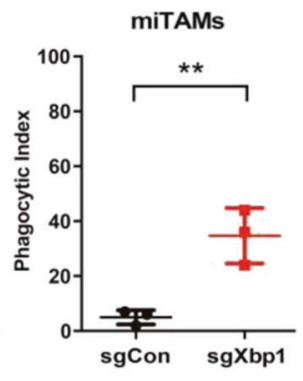

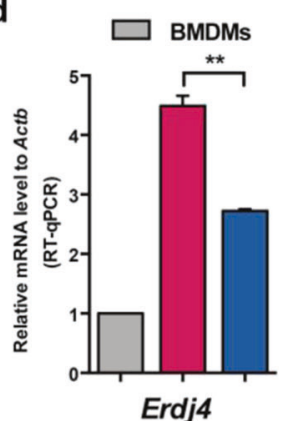

Erdj4

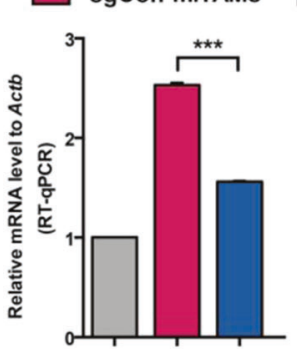

Thbs 1

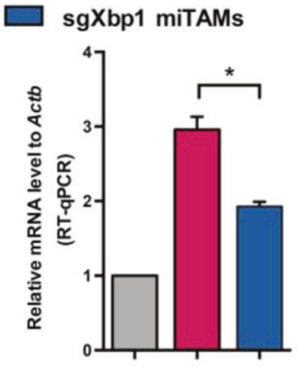

Sirpa

GSE86048
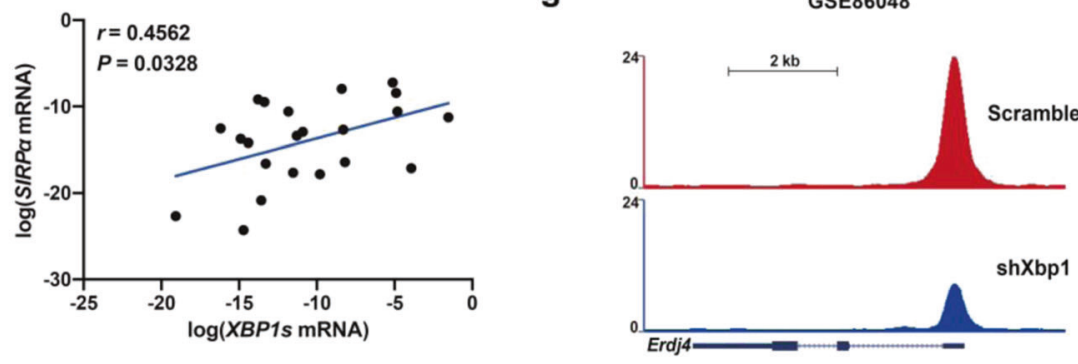

GSE68468
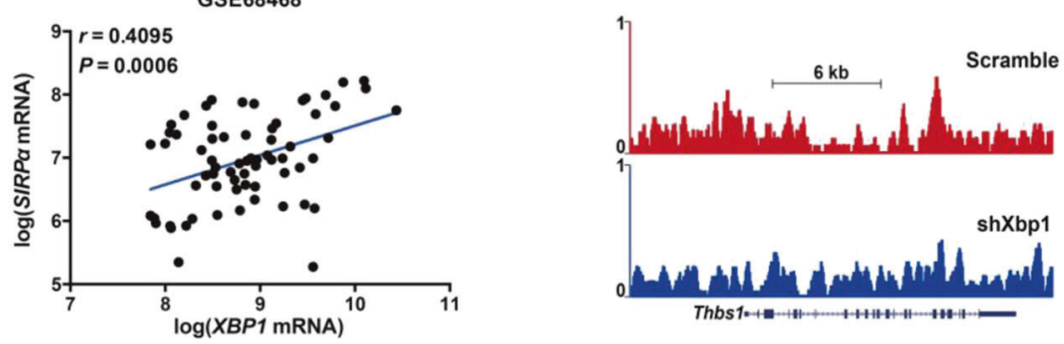

h
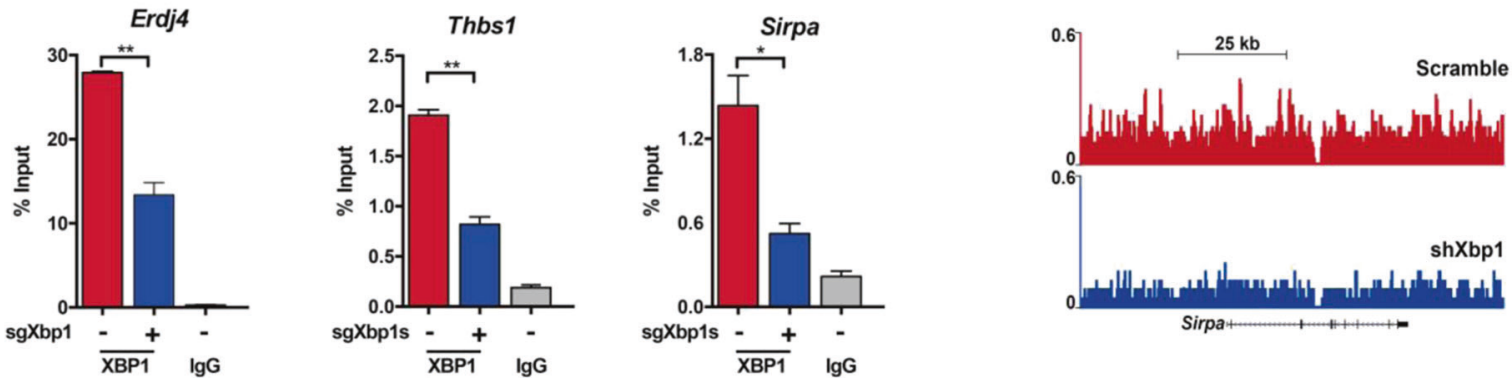
Fig. 5 Effect of XBP1 on macrophages phagocytosis. a Representative images of phagocytosis assays using RFP-labeled human CRC cell, DLD1cells (DLD1-RFP) and sgCon or sgXBP1 hiTAMs $(n=3)$. Yellow arrows denote phagocytic events. Scale bar $=200 \mu \mathrm{m}$. $\mathbf{b}$ Representative images of phagocytosis assays using RFP-labeled mouse CT26 cells (CT26-RFP) and sgCon or sgXbp1 miTAMs $(n=3)$. Yellow arrows denote phagocytic events. Scale bar $=200 \mu \mathrm{m}$. c Results of phagocytosis assays of the two groups in $\mathbf{a}, \mathbf{b}$. ${ }^{* *} P<0.01 ; t$-test. d Relative mRNA levels of $X B P 1$ and phagocytosis-associated genes in BMDM, sgCon miTAMs and sgXbp1 miTAMs, validated by RT-qPCR. Bars represent mean \pm SD of three experimental replicates. ${ }^{*} P<0.05$, ${ }^{*} P<0.01,{ }^{* *} P<0.001 ; t$-test. e Expression of THBS1 and SIRPa versus XBP1 in TAMs sorted from CRC patients $(n=27$ total). $r$, Spearman's rank correlation test. $\mathbf{f}$ Correlation of XBP1 with THBS1 and SIRPa in CRC patients. The association was analyzed using coefficient measures of the linear relationships in the public GEO database (GSE68468). $\mathbf{g}$ Track view of Erdj4, Thbs 1, and Sipra ChIP-seq density upon silencing of Xbp1 in the ChIP-seq online database (GSE86048). $\mathbf{h}$ ChIP-qPCR experiments measuring XBP1 binding on Erdj4, Thbs1, and Sipra segments. Bars represent mean \pm SD of three experimental replicates. ${ }^{*} P<0.05,{ }^{* *} P<0.01$. $P$-values were determined using $t$-test

that abrogating XBP1 function in TAMs could reduce the expression of tumor-promoting cytokines and also inhibit the "don't eat me" signals of macrophages in vivo. Together, these data indicated that targeting XBP1 in TAMs inhibit the progression of CRC.

\section{DISCUSSION}

We developed a potential strategy for CRC immunotherapy via the modulation of XBP1 activation in TAMs associated with CRC. XBP1 depletion in TAMs changed the cytokine expression patterns and disrupted the self-recognition capacity of TAMs, thereby enhancing the anti-tumor activity of macrophages, which led to the inhibition of tumor progression.

UPR activation endows malignant cells with greater tumorigenic, metastatic, and drug resistant capacity. ${ }^{17}$ XBP1 is overexpressed in colon cancer cells, whereas it was found to be unreactive in normal colon epithelial cells. ${ }^{34}$ In tumor microenvironment, nutrient deprivation, oxygen limitation, high metabolic demand, and oxidative stress, disturb the protein-folding capacity of the ER, thereby provoking a cellular state of ER stress. ${ }^{17,35,36}$ The IRE1a-XBP1 pathway may reasonably be considered a target candidate for cancer treatment. However, targeting ER stress in cancer cells has not yielded satisfactory results. Inhibiting IRE1 RNase activity in patientderived xenograft models, using small molecule inhibitor 8866, restrained MYC-overexpressing tumor growth but did not reduce tumors expressing $\mathrm{MYC}^{37}$ In $\mathrm{APC}^{\mathrm{min} /+}$ mouse model, epithelialspecific XBP1 deficiency was associated with a profound increase in tumorigenesis in CAC. ${ }^{38}$ The current study determined that XBP1 was present in both cancer cells and hTAMs sorted from CRC samples, whereas the amount of XBP1 present in TAMs was significantly higher than that present in cancer cells. Knockout of XBP1 in TAMs significantly inhibited the progression and metastasis of colon cancer. Thus, XBP1 activation in TAMs, but not in cancer cells, might be a potential immunotherapeutic target for CRC.

Shan et al. reported that IRE1 a promotes macrophage polarization to M1 in white adipose tissue. They showed that IRE1a downregulates Irf4 and KIf4 expression and suppresses M2 polarization, through a mechanism that requires its RNase activity, but presumably not its Xbp1 mRNA splicing activity. ${ }^{23}$ However, our results showed that XBP1 could upregulate the protumorigenic cytokines expression and reduce the phagocytosis, which could enhance the pro-tumorigenic function of macrophages (M2-like TAMs) in the tumor microenvironment. Therefore, whether ER stress promotes macrophage polarization to the M1 or M2 phenotype depends on the exact inflammation or tumor microenvironment stimulation in which the macrophage are located at that time.

The pro-tumor function of TAMs is executed by expressing cell surface receptors, cytokines, chemokines, and enzymes that activate Treg cells or suppress other effector cells. ${ }^{25}$ Therefore, we investigated whether XBP1 signaling contributes to the expression signature of TAMs. Inhibition of XBP1 in TAMs downregulated the expression of pro-tumor cytokines, such as IL-4, IL-6, MMP2 and VEGFA. This converted TAMs from a pro-tumor function to an anti-tumor function.
As a transcriptional factor, XBP1 regulates the expression of cytokines in response to stimuli, ${ }^{20}$ binds directly to the promoter regions of IL-6 and VEGFA and activates its expression, ${ }^{39,40}$ and this observation was also confirmed by our study. Bevacizumab (therapeutic anti-VEGF antibodies) is used, in combination with chemotherapy or targeting therapy, to clinically treat colon cancer. ${ }^{41}$ IL-6 is the key component of the cytokine release syndrome in immunotherapy-induced adverse events, and combination of IL-6 antibody (or IL-6R antibody) is now widely used in CAR-T cell therapy. ${ }^{42}$ Therefore, these results may provide a theoretical basis suggesting that targeting XBP1 alone might be an alternative strategy to targeting a combination of IL- 6 and VEGFA for CRC therapy.

Besides secreting soluble cytokines, macrophages participate in eliminating tumors via phagocytosis. ${ }^{43}$ Macrophage-based phagocytosis relies on the recognition of "eat me" or "don't eat me" signals emanating from target cells. Cancer cell surfaces express "don't eat me" signals and interact with recognition molecules on the surface of macrophages. Such interactions result in the blockage of phagocytosis. These signals include CD47/SIRPa, ${ }^{29}$ PDL1/PD- $1,{ }^{44}$ CD24/Siglec-10, ${ }^{45}$ and MHC-I/LILRB1. ${ }^{46}$ We found that XBP1 overexpression dramatically decreased phagocytosis by TAMs, but XBP1 knockout increased the phagocytic capacity of TAMs. Moreover, inhibition of XBP1 significantly reduced the expression of SIRPa and THBS1 in TAMs. ChIP results indicated that XBP1 directly regulates the transcription of SIRPa and THBS1. Our study reveales a new mechanism of XBP1 in TAMs that promotes phagocytosis of tumor cells by disrupting self-recognition.

CRC exhibits an immunosuppressive microenvironment similar to the other cancers. Immunotherapies that utilize PD-1/PD-L1 antibodies and CTLA-4 are currently undergoing clinical trials with some encouraging results. ${ }^{47}$ But the benefits seen in CRC have been limited to a few groups of patients with MSI-H tumors, which makes up only $3.5-17 \%$ of all CRCs. ${ }^{48,49}$ Therefore, a new therapeutic approach that does not only benefit a select group of CRC patients is highly desired. First, we treated CRC in AOM-DSS mouse model with clodrolip and found that the effect was not significant. This could be due to the depletion of all macrophages, including both the "M1" and "M2" macrophages by clodrolip. Thus, selectively diminishing the tumor-promoting function of TAMs might be a better strategy. Targeting TAM inhibitory ("do not eat") signals-CD47/SIRPa axis by therapeutic SIRPa antibodies could inhibit tumor formation inAOM-DSS-induced CRC. However, our results showed that targeting XBP1 in TAMs were more effective due to its dual function of inhibiting tumor-promoting cytokines and recognition signals. Furthermore, targeting XBP1 in TAMs inhibited the expression of PD-L1, ${ }^{50}$ SIRPa, and THBS1, together with the blockage of cytokines, simulated the effects of a powerful combination of several popular therapeutic programs. AAV2-sgXbp1, which conditionally deletes XBP1 in TAMs, dramatically inhibited tumor formation in AOM-DSS mouse model.

XBP1 activation is detected in TAMs of colon cancer patients and AOM-DSS mouse model in our study. XBP1 activation enhances the pro-tumor function of TAMs. Targeting XBP1 inhibits the ability of pro-tumor cytokines, such as IL-6 and VEGFA, as well as promotes tumor growth and metastasis. Concurrently, XBP1 inhibition 
downregulates the expression of membrane proteins, SIRPa and THBS1, blocking the "don't eat me" recognition signal, enhancing the phagocytosis function (Fig. 7). Although more evidence of the therapeutic effect of XBP1 signaling in TAMs should be accumulated, our results suggest that XBP1 pathway in TAMs shows potential as a novel therapeutic target in CRC treatment.

\section{MATERIALS AND METHODS}

Tissues, mice, and cell lines

Human CRC specimen samples were procured from the Surgical Pathology Unit at the Chinese Academy of Medical Sciences Cancer Hospital (Beijing, China). Informed consent was obtained from all subjects, and this study was approved by the ethical
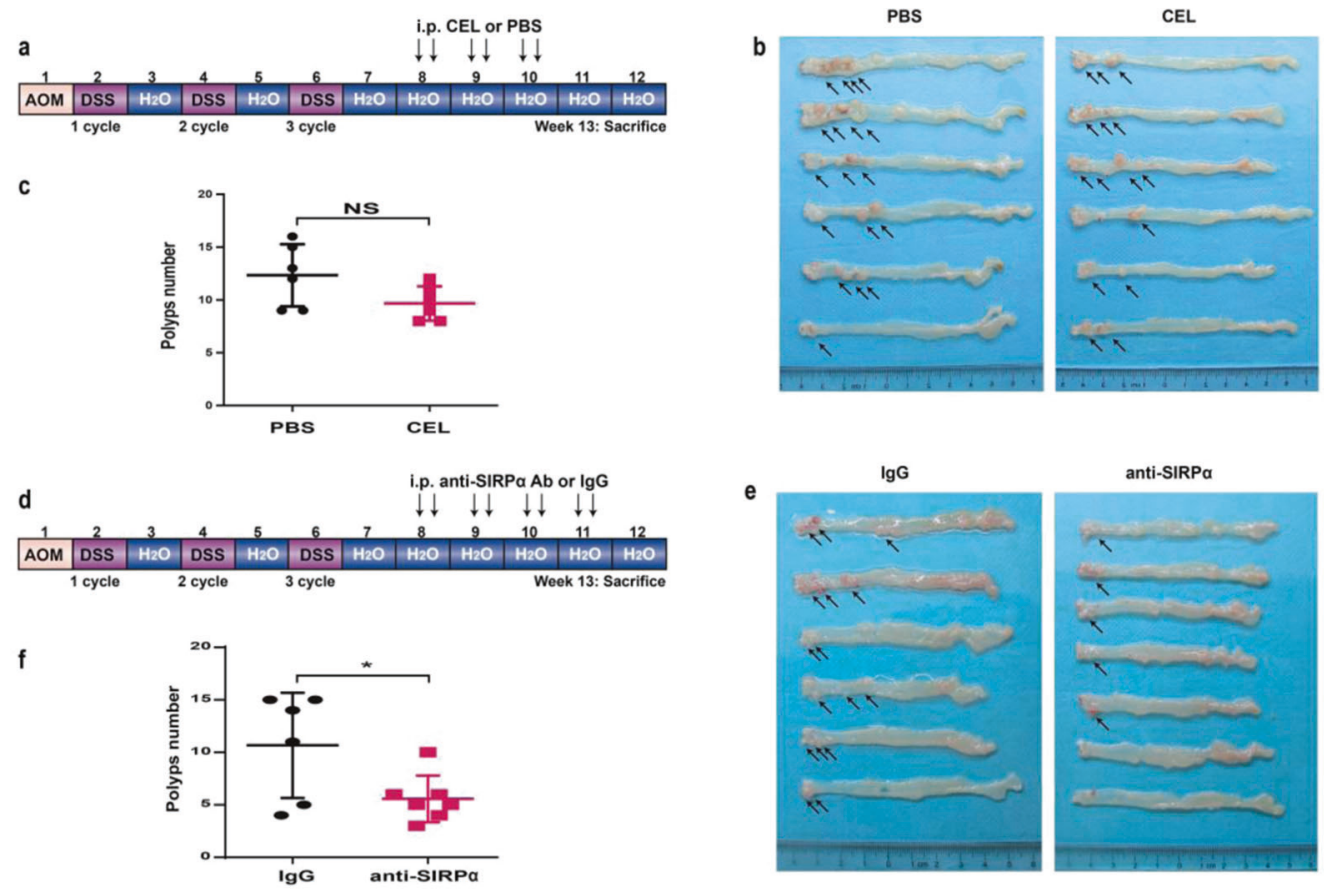

g
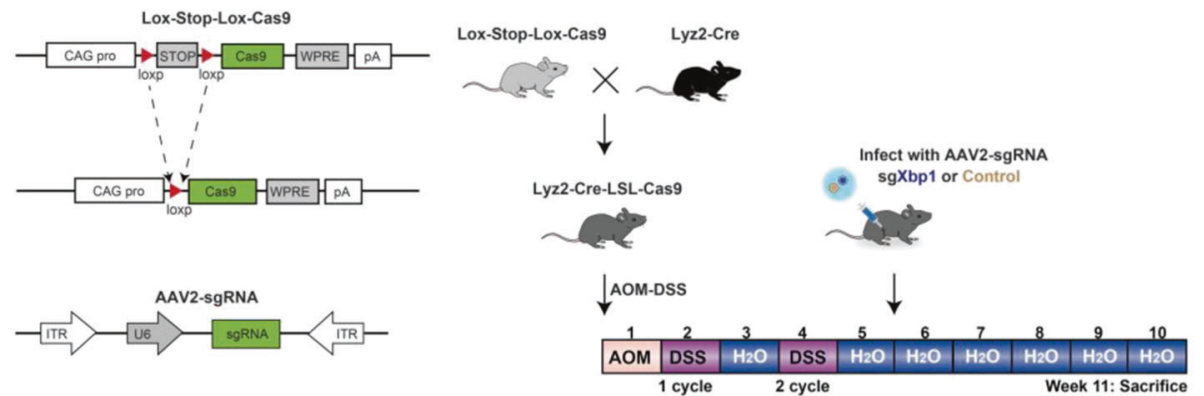

h

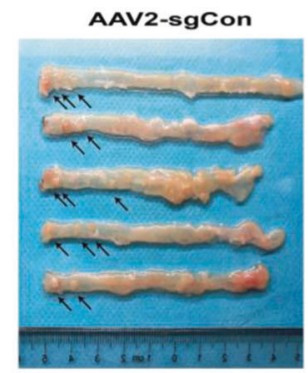

j

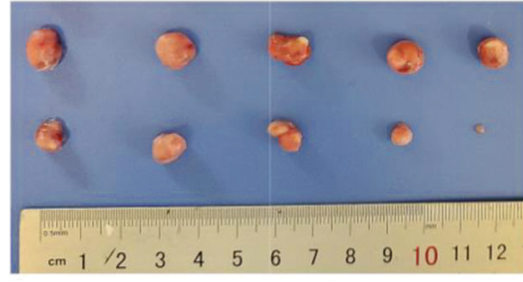

PDX+ sgCon hiTAMs

PDX+

sgXbp1 hiTAMs

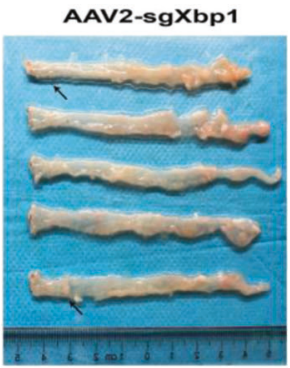

i
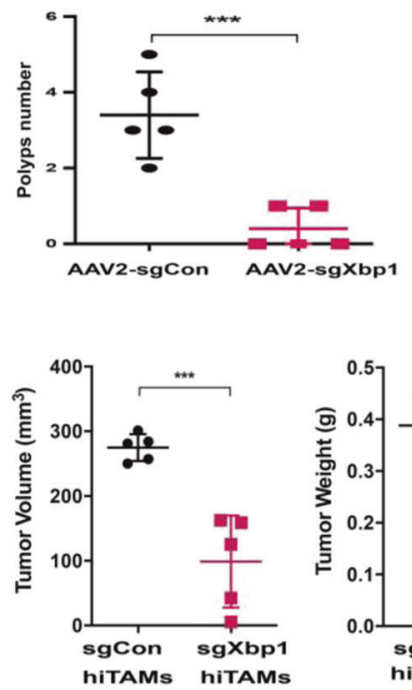

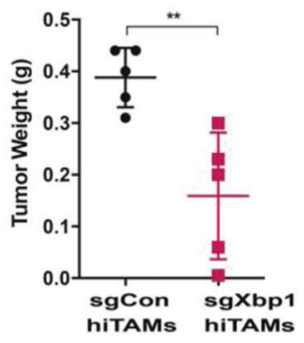


Fig. 6 Therapeutic effects of targeting UPR/ER-XBP1 signaling in TAMs. a Schematic overview of macrophage depletion in an AOM-DSS model. b Pictures of the whole colons. The arrowhead indicates macroscopic polyps. c Mean macroscopic polyp number in whole colons. d Schematic overview of the administering of anti-SIRP $\alpha$ antibodies during late stages of the AOM-DSS model. Mice were treated with antiSIRP $\alpha$ antibodies $(8 \mathrm{mg} / \mathrm{kg}$ ) vs. control lgG twice/week after the third DSS cycle for 4 weeks. Colons were removed at week 13 following AOM injection. e Pictures of the whole colon. The arrowhead indicates macroscopic polyps. $\mathbf{f}$ Mean macroscopic polyp numbers in whole colons ( $n=6$ for IgG and $n=7$ for anti-SIRP $\alpha$ Ab). $g$ Schematic representing the generation of mice with genetically targeted and deficient XBP1 in TAMs. Mice were treated with AAV2-sgXbp1 $\left(5 \times 10^{11} /\right.$ mouse, i.p.) vs. control AAV2-sgCon twice/week after the second DSS cycle for 4 weeks. Colons were removed at week 11 after AOM injection. $\mathbf{h}$ Pictures of the whole colon. The arrowhead indicates macroscopic polyps. i Mean macroscopic polyp number in whole colons $\left(n=5\right.$ for sgCon and $n=5$ for sgXbp1). NS $=P>0.05,{ }^{*} P<0.05,{ }^{* * *} P<0.001$. $P$-values were determined using $t$-test. $\mathrm{j}$ Representative photograph showing tumor formation in NOD/SCID mice injected s.C. with CRC PDX and two groups of TAMs: PDX + sgCon hiTAMs; and PDX + sgXBP1 hiTAMs. k Tumor volumes and tumor weights were resected and measured 3 weeks later. ${ }^{* *} P<0.01,{ }^{* * *} P<0.001 ;$ Mann-Whitney $U$-test

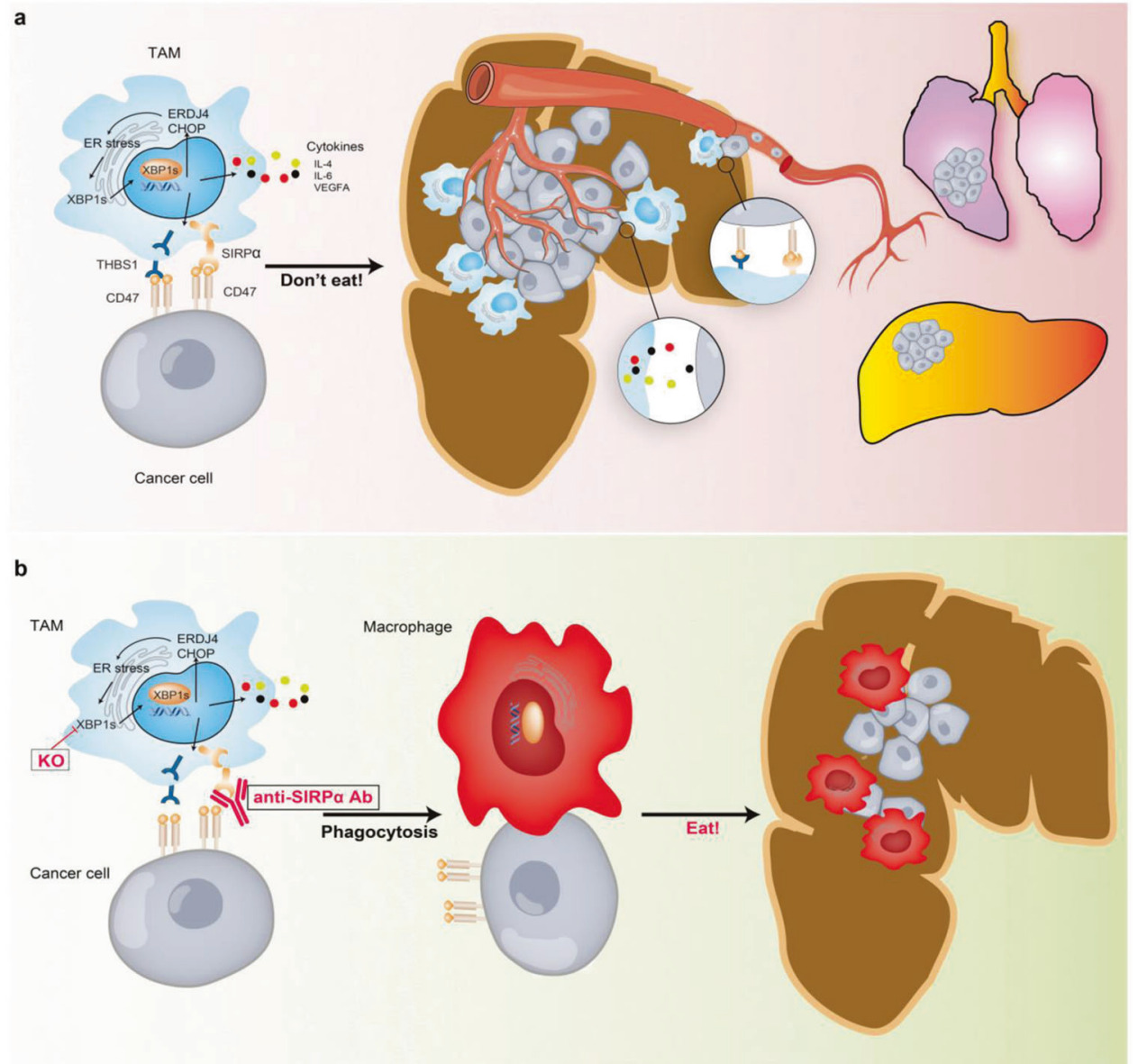

Fig. 7 Scheme depicting the contribution of XBP1 in TAMs to colon cancer progression. a In the tumor microenvironment, activation of UPR/ ER-XBP1 signaling in TAMs induced the production of cytokines, which inhibited macrophage phagocytosis of tumor cells via the disruption of self-recognition. Therefore, TAMs promote the metastasis of colorectal cancer. b Disabling UPR/ER-XBP1 signaling or treatment with antiSIRP $\alpha$ antibodies may enhance anti-cancer capacity in a harsh tumor microenvironment

committees of the Cancer Hospital, Chinese Academy of Medical Sciences. The clinical backgrounds for these patients are shown in Supplementary Tables S1-S3. Peripheral blood was collected from patients and centrifuged with Ficoll density gradient, following which the middle layer cells were gathered for fluorescenceactivated cell sorting (FACS) analysis. Mice were housed at our Institutional Animal Care unit. All animal experiment protocols were approved by the ethical committee of the Chinese Academy of Medical Sciences, Cancer Hospital.

THP-1 (TIB-202, RRID:CVCL_0006), HCT116 (CCL-247, RRID: CVCL_0291) and DLD1 (CCL-221, RRID:CVCL_0248) cells were from ATCC. Ana-1 (EP-CL-0023, RRID:CVCL_0142) and CT26 cells (EP-CL0071, RRID:CVCL_7254) were from Elabscience (Texas, USA). All the cells were maintained in RPMI 1640 (Bioroc ${ }^{\mathrm{TM}}$, China), supplemented with $10 \%$ heated-inactivated fetal bovine serum, $100 \mathrm{UI} / \mathrm{mL}$ penicillin, and $100 \mu \mathrm{g} / \mathrm{mL}$ streptomycin.

\section{AOM-DSS-induced CRC model}

On day 1, C57BL/6J mice were injected i.p. with a single dose of AOM (Sigma-Aldrich Corp., St. Louis, MO, USA) at $2.5 \mathrm{mg} / \mathrm{kg}$ body weight as previously described. ${ }^{51}$ One week later, these mice were administered three cycles of $2.5 \%$ DSS for 5 days in sterile water, followed by one week in regular sterile water. Starting at week 8 , the mice received different treatments (CEL clodronate liposome or anti-SIRPa). Tumor induced mice were randomized into respective treatment groups. CEL or PBS was administered twice 
12

a week (i.p., $12.5 \mu \mathrm{g}$ ). Anti-SIRPa (Bio X Cell, clone P84) or IgG were administered twice a week (i.p.). The mice were sacrificed 13 weeks later, colons removed and cut longitudinally. The number of tumors in colon of each mouse was counted. Portions of the distal colon were fixed with $4 \%$ paraformaldehyde overnight at $4{ }^{\circ} \mathrm{C}$ and embedded in paraffin.

Tumor-associated macrophage induction and lentivirus hTAMs $\left(C D 14^{+} \mathrm{CD}_{11 \mathrm{~b}^{+}} \mathrm{CD} 206^{+}\right)$and PBMs $\left(\mathrm{CD} 14^{+} \mathrm{CD} 11 \mathrm{~b}^{+}\right)$in single-cell suspensions were sorted using FACSCalibur flow cytometry (Becton, Dickinson and Co., San Jose, CA, USA). Antihuman CD14 (clone 63D3, labeled with Percp-cy5.5), CD11b (clone ICRF44, labeled with APC) and CD206 (clone 15-2, labeled with PE) were purchased from BioLegend (San Diego, CA). mTAMs (CD14

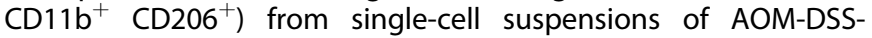
induced colon tumors were sorted. APC-anti-mouse CD11b (clone M1/70) was from eBioscience (CA, USA). Percp-cy5.5-anti-mouse CD14 (clone Sa14-2) and FITC-anti-mouse CD206 (clone C068C2) were from BioLegend. Control spleen macrophages (sMs) from spleens of naive or CRC-bearing mice were FACS sorted by CD14 and $\mathrm{CD} 11 \mathrm{~b}$. In all cases, BMDMs were generated from bone marrow in C57BL/6J mice via incubation in media supplemented with $20 \mathrm{ng} / \mathrm{mL}$ recombinant granulocyte macrophage colonystimulating factor (M-CSF) (R\&D, MN, USA). BMDMs were cultured in DMEM supplemented with $10 \%$ FBS at $37^{\circ} \mathrm{C}$ in $5 \% \mathrm{CO} 2$ and harvested on day 7 of expansion and used for subsequent $C M$ induce. $^{52}$ After $72 \mathrm{~h}$, cells were detected for FACS to confirm $\mathrm{CD}^{206^{+}}$macrophages (miTAMs). PBMC-derived macrophages were generated from peripheral blood mononuclear cell and were prepared to incubate with conditioned medium for $72 \mathrm{~h}$. $\mathrm{CD}^{2} 6^{+}$macrophages were considered to be hiTAMs.

THP-1 (M0) cells were generated from THP-1 via incubation with LPS (10 ng/mL) for $96 \mathrm{~h}$. THP-1 (M2) cells were generated from THP-1 (M0) incubated with IL-4 (20 ng/mL) for $48 \mathrm{~h}$. TAMs (THP-1 CM) were generated from THP-1 (M2) cells induced via exposure to condition medium from HCT116 cells for $72 \mathrm{~h}$. SgXBP1 hTAMs were inoculated with lentivirus LentiCRISPR v2 sgRNA targeting XBP1 (5'-GGGCATTGAAGAACATGAC-3'), and sgCon hTAMs were inoculated with a nontargeting sgRNA (5'-ACGGAGGCTAAGCGTCGCAA-3').

TAMs (Ana-1 CM) were generated from Ana-1 cell induced by exposure to condition medium from CT26 cells for $72 \mathrm{~h}$. SgXbp1 mTAMs were inoculated with lentivirus LentiCRISPR v2 sgRNA targeting Xbp1 (5'-ACTTGTCCAGAATGCCCAAA-3'), and sgCon mTAMs were inoculated with a nontargeting sgRNA (5'-GCGAGGTATTCGGCTCCGCG-3').

RNA-seq and macrophage transcriptional profile

TAMs were sorted from human CRC tumor and AOM-DSS-induced CRC mice single-cell suspensions. Total RNA was isolated using RNeasy Mini Kit (Qiagen, Germany) and RNA quality and integrity confirmed via an Agilent Bioanalyzer 2100. Next, mRNA libraries were generated and sequenced at the WuXiNextCODE Genomics (Shanghai) Co., Ltd.

\section{Immunofluorescence staining}

Tumor tissues were fixed in $4 \%$ paraformaldehyde and immunofluorescence was performed on 5 - $\mu$ m-thick paraffin sections after heat-induced antigen retrieval. The following primary antibodies were used: CD68 (1:200, Abcam, ab53444); CD206 (1:200, Proteintech, 60143-1-lg), XBP1 (1:100, Abcam, ab37152), F4/80 (1:200, Abcam, ab6640) and EPCAM (1:800, CST, \#2929). Subsequently, we incubated the slides with fluorescent secondary antibodies (Fluorescein (FITC)-conjugated Goat Anti-Rabbit $\lg \mathrm{G}(\mathrm{H}$ $+\mathrm{L})$, 1:1000, Proteintech, SA00003-2; Rhodamine (TRITC)-conjugated Goat Anti-Rat IgG $(\mathrm{H}+\mathrm{L})$, 1:1000, Proteintech, SA00007-7; Alexa Fluor ${ }^{\circledR} 647$ Conjugate Goat Anti-mouse lgG $(H+L), 1: 1000$, Cell Signaling Technology, \#4410), DAPI (Thermo Scientific,
\#62247) and Opal 7-Color Manual IHC Kit (Akoya Biosciences, NEL811001KT) for multilabel immunofluorescence analysis.

\section{Phagocytosis assay}

sgCon or sgXBP1 TAMs $\left(5 \times 10^{4}\right)$ were co-cultured with RFPlabeled DLD1 or CT26 cancer cells $\left(2.5 \times 10^{5}\right)$ in 24-well tissueculture plates. Living cells were assessed by UltraVIEW VoX (PerkinElmer) for $8 \mathrm{~h}$ at $37^{\circ} \mathrm{C}$ and wells were washed thoroughly with DMDM three times and analyzed using Volocity software (PerkinElmer). Phagocytic index was calculated using as described, phagocytic index = number of ingested cells/(number of macrophages/100). ${ }^{53}$

Orthotopic xenograft CRC mouse model, liver metastasis, and lung metastasis model

For the orthotopic xenograft CRC mouse model, CT26 $\left(2 \times 10^{5}\right.$ cells) were mixed with the indicated miTAMs $\left(2 \times 10^{5}\right.$ cells $)$ and were co-injected into the wall of the cecum in NOD/SCID mice ( $n=5$ per group). After 4 weeks, all the mice were sacrificed. Colons and livers, were harvested to assess the tumor burden. HE staining demonstrating the histology of tumors formed in the livers was observed using the Imagescope software as described. ${ }^{54}$

NOD/SCID mice were injected intrasplenically with CT26luciferase cells $\left(1 \times 10^{5}\right.$ cells, stably expressed firefly luciferase) and miTAMs ( $1 \times 10^{5}$ cells) by splenectomy 3 min after injections, for the liver metastasis model..$^{55}$ On day 15 following model establishment, $200 \mu \mathrm{L}$ of $15 \mathrm{mg} / \mathrm{mL}$ luciferin (PerkinElmer) was intraperitoneally injected into anesthetized mice, and bioluminescence was examined 10 min after injection using an IVIS Lumina system (PerkinElmer). Bioluminescence was quantified based on the photon flux ratio. HE staining demonstrating the histology of tumors formed in the livers with the ImageScope software.

For the induction of lung metastasis, NOD/SCID mice were injected intravenously (i.v.) with CT26 cells $\left(1 \times 10^{5}\right.$ cells) and miTAMs $\left(1 \times 10^{5}\right.$ cells $)$ in $100 \mu \mathrm{L}$ PBS, and were sacrificed 20 days later. To quantify the metastatic burden, pulmonary metastatic nodule numbers were calculated. HE staining demonstrating the histology of tumors formed in the lungs was observed using the ImageScope software.

\section{Cytokine antibody array}

CM from sgCon TAMs and paired sgXBP1 TAMs were tested for cytokine secretion using selected cytokine antibody arrays, following the manufacturer's instructions (Ray Biotech, Inc, Norcross, GA, USA).

\section{ChIP-qPCR}

BMDM cells were separated from bone marrow of C57BL/6J mice and trained to produce TAMs using condition medium from CT26 cells. Cells were washed twice in cold PBS supplemented with protease inhibitors (Roche), fixed for $15 \mathrm{~min}$ in 1\% formaldehyde (Sigma-Aldrich) at room temperature and quenched by adding $125 \mathrm{mmol} / \mathrm{L}$ glycine for $5 \mathrm{~min}$. ChIP was performed with XBP1 antibodies (sc-7160; Santa Cruz Biotechnology, CA). All primers used are described in Supplementary Table S3.

Generation of macrophages-specific Cre-dependent Cas9 mice Conditional Cas9 mice Lox-stop-Lox-Cas9 (C57BL/6-Gt(ROSA) 26Sor ${ }^{\text {tm 1(CAG-LNL-Cas9)Smoc) }}$ were obtained from the Shanghai Model Organisms Center, Inc. and crossed with bone marrow cellsspecific Lyz2-Cre mice (C57BL/6-Lyz2 ${ }^{\text {em1(2A-CreERT2-WPRE-pA)Smoc }) ~ i n ~}$ order to generate experimental Lyz2-Cre-LSL-Cas9 mice. The following primers were used to genotype Cas9: Cas9_Common-S 5'-TCCCGACAAAACCGAAAATCTGTGG-3'; Cas9_Wild-AS 5'-GGGG CGTGCTGAGCCAGACCTCCAT-3'; Cas9_Mut-AS 5'- TGCATCGCATTG TCTGAGTAGG-3') and Lyz2-Cre (5'-CTTGGGCTGCCAGAATTCTC-3' for common-S; 5'-CCCAGAAATGCCAGATTACG-3' for Wild-type-AS; 
and 5'-TTACAGTCGGCCAGGCTGAC-3' for Cre-AS in Lyz2-Cre mice as previously described. ${ }^{56,57}$

SgRNA-Xbp1 Adeno-associated virus (AAV2) production Effective sgRNAs targeting mouse XBP1 were first validated using lentiviral vectors, and adenovirus pX601-AAV-CMV::NLS-SaCas9-NLS3xHA-bGHpA;U6::Bsal-sgRNA (Addgene) targeting (AAV2-sgXbp1) or nontargeting XBP1 (AAV2-sgControl) were generated. Then, 293FT cells were transfected using Lipofectamine 2000 (Invitrogen, Carlsbad, CA, USA) with the AAV vector along with helper packaging vectors. Two days later, the cells and media were collected and subjected to four freeze-thaw cycles by alternating between an ethanol dry ice bath and $37^{\circ} \mathrm{C}$. Cell debris was removed by centrifugation and the supernatant was collected, passed through a $0.45 \mu \mathrm{m}$ filter, aliquoted, and frozen at $-80^{\circ} \mathrm{C}$ until further use.

Quantification and statistical analysis

All experiments were repeated at least twice, where the results of repeats were similar. Animal experiments used between three and six mice per group. Statistical significance was set at $P<0.05$. All statistical analyses were conducted using Graph Pad Prism 7.0. Differences between the means of experimental groups were analyzed using unpaired Student's $t$-test or ANOVA analysis. Error bars represent SEM of independent samples assayed within representative experiments. Survival rates were compared using the log-rank test. All survival experiments used at least six mice per group. This number provides a $5 \%$ significance level and $95 \%$ power to detect differences in the survival of $20 \%$ or greater.

\section{DATA AVAILABILITY}

All the datasets used and/or analyzed during this study are available from the corresponding author on reasonable request.

\section{ACKNOWLEDGEMENTS}

We are grateful to Dr. Xiaoyu Hu (Tsinghua University, China) for kindly providing Lyz2 Cre mice and technique direction. We thank Dr. Lan Kang (Tsinghua University, China) for isolation methods of resident peritoneal macrophages. We appreciate WuXiNextCODE Genomics (Shanghai) Co., Ltd for the RNA-seq analysis. This work was supported by the National Natural Science Foundation of China (81772638 and 81903025) and CAMS Innovation Fund for Medical Sciences (2016-I2M-1-001).

\section{AUTHOR CONTRIBUTIONS}

Y.Z. designed, conducted the experiments, and drafted the manuscript. H.Z. and N.X. supervised the study, designed the experiments, and edited the manuscript. W.Z. and Y.L. performed in vitro experiments. M.H. performed C57BL/6J-AOM-DSS model and analyzed data. X.L. and Y.W. performed in vivo experiments and analyzed data. Z.Z and P.W. performed surgeries and provided all human patients specimens.

\section{ADDITIONAL INFORMATION}

Supplementary information The online version contains supplementary material available at https://doi.org/10.1038/s41392-021-00761-7.

Competing interests: The authors declare no competing interests.

\section{REFERENCES}

1. Lasry, A., Zinger, A. \& Ben-Neriah, Y. Inflammatory networks underlying colorectal cancer. Nat. Immunol. 17, 230-240 (2016).

2. Fridman, W. H., Pages, F., Sautes-Fridman, C. \& Galon, J. The immune contexture in human tumours: impact on clinical outcome. Nat. Rev. Cancer 12, 298-306 (2012).

3. Boland, P. M. \& Ma, W. W. Immunotherapy for colorectal cancer. Cancers (Basel) 9 , 50 (2017).

4. Gentles, A. J. et al. The prognostic landscape of genes and infiltrating immune cells across human cancers. Nat. Med. 21, 938-945 (2015).
5. Forssell, J. et al. High macrophage infiltration along the tumor front correlates with improved survival in colon cancer. Clin. Cancer Res. 13, 1472-1479 (2007).

6. Feng, Q. et al. Tumor-associated macrophages as prognostic and predictive biomarkers for postoperative adjuvant chemotherapy in patients with stage II colon cancer. Clin. Cancer Res. 25, 3896-3907 (2019).

7. Sica, A. \& Mantovani, A. Macrophage plasticity and polarization: in vivo veritas. J. Clin. Invest. 122, 787-795 (2012).

8. Zhang, L. et al. Single-cell analyses inform mechanisms of myeloid-targeted therapies in colon cancer. Cell 181, 442-459 e429 (2020).

9. Qian, B. Z. \& Pollard, J. W. Macrophage diversity enhances tumor progression and metastasis. Cell 141, 39-51 (2010).

10. Tariq, M. et al. Macrophage polarization: anti-cancer strategies to target tumorassociated macrophage in breast cancer. J. Cell Biochem. 118, 2484-2501 (2017).

11. Mantovani, A., Marchesi, F., Malesci, A., Laghi, L. \& Allavena, P. Tumour-associated macrophages as treatment targets in oncology. Nat. Rev. Clin. Oncol. 14, 399-416 (2017).

12. Urra, H., Dufey, E., Avril, T., Chevet, E. \& Hetz, C. Endoplasmic reticulum stress and the hallmarks of cancer. Trends Cancer 2, 252-262 (2016).

13. Walter, P. \& Ron, D. The unfolded protein response: from stress pathway to homeostatic regulation. Science 334, 1081-1086 (2011).

14. Dong, $H$. et al. The IRE 1 endoplasmic reticulum stress sensor activates natural killer cell immunity in part by regulating c-Myc. Nat. Immunol. 20, 865-878 (2019).

15. Bettigole, S. E. et al. The transcription factor XBP1 is selectively required for eosinophil differentiation. Nat. Immunol. 16, 829-837 (2015).

16. Iwakoshi, N. N., Pypaert, M. \& Glimcher, L. H. The transcription factor XBP-1 is essential for the development and survival of dendritic cells. J. Exp. Med. 204, 2267-2275 (2007).

17. Cubillos-Ruiz, J. R. et al. ER Stress Sensor XBP1 Controls Anti-tumor Immunity by Disrupting Dendritic. Cell Homeost. Cell 161, 1527-1538 (2015).

18. Song, M. et al. IRE1alpha-XBP1 controls $\mathrm{T}$ cell function in ovarian cancer by regulating mitochondrial activity. Nature 562, 423-428 (2018).

19. Martinon, F., Chen, X., Lee, A. H. \& Glimcher, L. H. TLR activation of the transcription factor XBP1 regulates innate immune responses in macrophages. Nat. Immunol. 11, 411-418 (2010).

20. Kim, S. et al. Endoplasmic reticulum stress-induced IRE1alpha activation mediates cross-talk of GSK-3beta and XBP-1 to regulate inflammatory cytokine production. J. Immunol. 194, 4498-4506 (2015).

21. Oh, J. et al. Endoplasmic reticulum stress controls $M 2$ macrophage differentiation and foam cell formation. J. Biol. Chem. 287, 11629-11641 (2012).

22. Kapoor, N. et al. Transcription factors STAT6 and KLF4 implement macrophage polarization via the dual catalytic powers of MCPIP. J. Immunol. 194, 6011-6023 (2015).

23. Shan, B. et al. The metabolic ER stress sensor IRE1alpha suppresses alternative activation of macrophages and impairs energy expenditure in obesity. Nat. Immunol. 18, 519-529 (2017).

24. Xiu, F., Diao, L., Qi, P., Catapano, M. \& Jeschke, M. G. Palmitate differentially regulates the polarization of differentiating and differentiated macrophages. Immunology 147, 82-96 (2016).

25. Noy, R. \& Pollard, J. W. Tumor-associated macrophages: from mechanisms to therapy. Immunity 41, 49-61 (2014).

26. Batista, A. et al. IRE1alpha regulates macrophage polarization, PD-L1 expression, and tumor survival. PLoS Biol. 18, e3000687 (2020).

27. Miao, $\mathrm{H}$. et al. Macrophage ABHD5 promotes colorectal cancer growth by suppressing spermidine production by SRM. Nat. Commun. 7, 11716 (2016).

28. Li, R. et al. Macrophage-secreted TNFalpha and TGFbeta1 influence migration speed and persistence of cancer cells in $3 \mathrm{~d}$ tissue culture via independent pathways. Cancer Res. 77, 279-290 (2017).

29. Willingham, S. B. et al. The CD47-signal regulatory protein alpha (SIRPa) interaction is a therapeutic target for human solid tumors. Proc. Natl Acad. Sci. USA 109, 6662-6667 (2012).

30. Daubon, T. et al. Deciphering the complex role of thrombospondin-1 in glioblastoma development. Nat. Commun. 10, 1146 (2019).

31. Hiraoka, K. et al. Inhibition of bone and muscle metastases of lung cancer cells by a decrease in the number of monocytes/macrophages. Cancer Sci. 99, 1595-1602 (2008).

32. Feng, M. et al. Phagocytosis checkpoints as new targets for cancer immunotherapy. Nat. Rev. Cancer 19, 568-586 (2019).

33. Ring, N. G. et al. Anti-SIRPalpha antibody immunotherapy enhances neutrophil and macrophage antitumor activity. Proc. Natl Acad. Sci. USA 114, E10578-E10585 (2017).

34. Fujimoto, T. et al. Overexpression of human X-box binding protein 1 (XBP-1) in colorectal adenomas and adenocarcinomas. Anticancer Res. 27, 127-131 (2007). 
XBP1 regulates the protumoral function of tumor-associated macrophages in... Zhao et al.

14

35. Augustin, R. C., Delgoffe, G. M. \& Najjar, Y. G. Characteristics of the tumor microenvironment that influence immune cell functions: hypoxia, oxidative stress, metabolic alterations. Cancers (Basel) 12, 3802 (2020).

36. Cubillos-Ruiz, J. R., Bettigole, S. E. \& Glimcher, L. H. Tumorigenic and immunosuppressive effects of endoplasmic reticulum stress in cancer. Cell 168, 692-706 (2017).

37. Zhao, N. et al. Pharmacological targeting of MYC-regulated IRE1/XBP1 pathway suppresses MYC-driven breast cancer. J. Clin. Invest. 128, 1283-1299 (2018).

38. Niederreiter, L. et al. ER stress transcription factor Xbp1 suppresses intestinal tumorigenesis and directs intestinal stem cells. J. Exp. Med. 210, 2041-2056 (2013).

39. Chen, C. \& Zhang, X. IRE1alpha-XBP1 pathway promotes melanoma progression by regulating IL-6/STAT3 signaling. J. Transl. Med. 15, 42 (2017).

40. Pereira, E. R., Liao, N., Neale, G. A. \& Hendershot, L. M. Transcriptional and posttranscriptional regulation of proangiogenic factors by the unfolded protein response. PLoS ONE 5, e12521 (2010).

41. You, X. H. et al. Chemotherapy plus bevacizumab as an optimal first-line therapeutic treatment for patients with right-sided metastatic colon cancer: a metaanalysis of first-line clinical trials. ESMO Open 4, e000605 (2020).

42. Chen, $\mathrm{H}$. et al. Management of cytokine release syndrome related to CAR-T cell therapy. Front. Med. 13, 610-617 (2019).

43. Feng, M. et al. Macrophages eat cancer cells using their own calreticulin as a guide: roles of TLR and Btk. Proc. Natl Acad. Sci. USA 112, 2145-2150 (2015).

44. Gordon, S. R. et al. PD-1 expression by tumour-associated macrophages inhibits phagocytosis and tumour immunity. Nature 545, 495-499 (2017).

45. Barkal, A. A. et al. CD24 signalling through macrophage Siglec-10 is a target for cancer immunotherapy. Nature 572, 392-396 (2019).

46. Barkal, A. A. et al. Engagement of MHC class I by the inhibitory receptor LILRB1 suppresses macrophages and is a target of cancer immunotherapy. Nat. Immunol. 19, 76-84 (2018).

47. Franke, A. J. et al. Immunotherapy for colorectal cancer: a review of current and novel therapeutic approaches. J. Natl Cancer Inst. 111, 1131-1141 (2019).

48. Gryfe, R. et al. Tumor microsatellite instability and clinical outcome in young patients with colorectal cancer. N. Engl. J. Med. 342, 69-77 (2000).

49. Llosa, N. J. et al. The vigorous immune microenvironment of microsatellite instable colon cancer is balanced by multiple counter-inhibitory checkpoints. Cancer Discov. 5, 43-51 (2015).
50. Liu, M. et al. MicroRNA-492 overexpression involves in cell proliferation, migration, and radiotherapy response of cervical squamous cell carcinomas. Mol. Carcinog. 57, 32-43 (2018).

51. Greten, F. R. et al. IKKbeta links inflammation and tumorigenesis in a mouse model of colitis-associated cancer. Cell 118, 285-296 (2004).

52. Lu, C. S. et al. Oct4 promotes M2 macrophage polarization through upregulation of macrophage colony-stimulating factor in lung cancer. J. Hematol. Oncol. 13, 62 (2020).

53. Jaiswal, S. et al. CD47 is upregulated on circulating hematopoietic stem cells and leukemia cells to avoid phagocytosis. Cell 138, 271-285 (2009).

54. Liu, H. et al. Mutant KRAS triggers functional reprogramming of tumor-associated macrophages in colorectal cancer. Sig. Transduct. Target. Ther. 6, 144 (2021).

55. Dupaul-Chicoine, J. et al. The Nlrp3 inflammasome suppresses colorectal cancer metastatic growth in the liver by promoting natural killer cell tumoricidal activity. Immunity 43, 751-763 (2015).

56. Ideno, N. et al. A pipeline for rapidly generating genetically engineered mouse models of pancreatic cancer using in vivo CRISPR-Cas9-mediated somatic recombination. Lab. Invest. 99, 1233-1244 (2019).

57. Coats, B. R. et al. Metabolically activated adipose tissue macrophages perform detrimental and beneficial functions during diet-induced obesity. Cell Rep. 20, 3149-3161 (2017)

(c) Open Access This article is licensed under a Creative Commons Attribution 4.0 International License, which permits use, sharing, adaptation, distribution and reproduction in any medium or format, as long as you give appropriate credit to the original author(s) and the source, provide a link to the Creative Commons license, and indicate if changes were made. The images or other third party material in this article are included in the article's Creative Commons license, unless indicated otherwise in a credit line to the material. If material is not included in the article's Creative Commons license and your intended use is not permitted by statutory regulation or exceeds the permitted use, you will need to obtain permission directly from the copyright holder. To view a copy of this license, visit http://creativecommons. org/licenses/by/4.0/.

(c) The Author(s) 2021 